\title{
High-frequency trading with fractional Brownian motion
}

\author{
Paolo Guasoni ${ }^{1}$. Yuliya Mishura ${ }^{2}$. \\ Miklós Rásonyi ${ }^{3}$
}

Received: 13 August 2019 / Accepted: 10 July 2020 / Published online: 8 October 2020

(C) The Author(s) 2020

\begin{abstract}
In the high-frequency limit, conditionally expected increments of fractional Brownian motion converge to a white noise, shedding their dependence on the path history and the forecasting horizon and making dynamic optimisation problems tractable. We find an explicit formula for locally mean-variance optimal strategies and their performance for an asset price that follows fractional Brownian motion. Without trading costs, risk-adjusted profits are linear in the trading horizon and rise asymmetrically as the Hurst exponent departs from Brownian motion, remaining finite as the exponent reaches zero while diverging as it approaches one. Trading costs penalise numerous portfolio updates from short-lived signals, leading to a finite trading frequency, which can be chosen so that the effect of trading costs is arbitrarily small, depending on the required speed of convergence to the high-frequency limit.
\end{abstract}

Keywords Fractional Brownian motion - Transaction costs · High frequency · Trading $\cdot$ Mean-variance optimisation

Guasoni is partially supported by SFI (16/IA/4443, 16/SPP/3347). Mishura is supported by the ToppForsk project Nr. 274410 of the Research Council of Norway with title STORM: Stochastics for Time-Space Risk Models. Rásonyi is supported by the NKFIH (National Research, Development and Innovation Office, Hungary) grant KH 126505 and by the "Lendület" grant LP 2015-6 of the Hungarian Academy of Sciences.

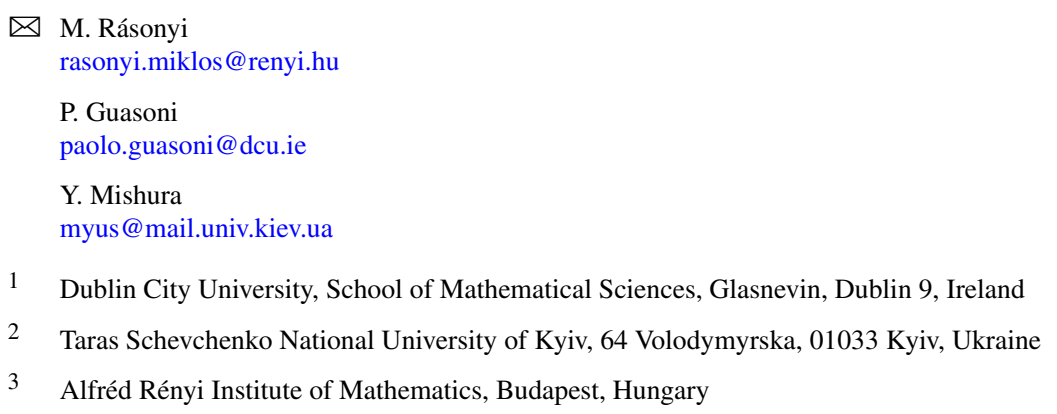




\title{
Mathematics Subject Classification (2010) 91G10 • 91G80
}

\author{
JEL Classification G11 · G12
}

\section{Introduction}

First proposed as a model of price dynamics by Mandelbrot [14], fractional Brownian motion $(\mathrm{fBm})$ has since puzzled researchers and stirred controversy for its elusive properties, which have confounded both empirical and theoretical work. Long-range dependence in asset prices, the property that originally motivated the use of $\mathrm{fBm}$ to describe price dynamics, remains undecided; see Greene and Fielitz [7], Fama and French [6], Poterba and Summers [17], Lo [13], Jacobsen [12], Teverovsky et al. [22], Willinger et al. [23], Baillie [1]. Arbitrage, which has plagued the adoption of fBm in models of optimal investment (Rogers [19], Salopek [20], Dasgupta and Kallianpur [5], Cheridito [2]) disappears with frictions (Guasoni [8], Guasoni et al. [10], Czichowsky and Schachermayer [4], Czichowsky et al. [3]), leading to finite expected profits; see Guasoni et al. [9].

This paper finds locally mean-variance optimal trading strategies in fractional Brownian motion and characterises their convergence and performance in the highfrequency limit. Our analysis starts from a fixed trading frequency, for which optimal strategies are directly proportional to the (conditionally) expected increment and inversely proportional to its variance. The central feature of fractional Brownian motion is that unlike diffusion models, the conditionally expected increment is not proportional to the length of the trading period, but to a power thereof - the Hurst exponent. Because the increment's standard deviation scales with the same power, the average Sharpe ratio is insensitive to the length of the trading period.

The key insight (Theorem 2.3) is that the high-frequency limit of such a forecast (the "latent drift" of fractional Brownian motion) is a white-noise process with a variance depending on the Hurst exponent, but invariant to any scaling of the process (which would equally scale both expected increments and their standard deviation). This result in turn leads to a cascade of implications for optimal continuous trading of fractional Brownian motion.

First, the optimal mean-variance performance from trading fractional Brownian motion is proportional to the length of the whole trading horizon - as for Brownian motion with drift - in spite of the different scaling of mean and variance on individual periods. The reason is that the cumulative performance of high-frequency trading fractional Brownian motion on a finite horizon is essentially equivalent to the average performance of a discrete-time model with infinitely many periods and independent, identically distributed Sharpe ratios. Both performances are deterministic because randomness disappears through ergodicity.

Second, the resulting performance is asymmetric in the Hurst exponent (Fig. 1 and Theorem 2.2), remaining bounded as the process approaches a white noise (near $H=0$ ), but diverging as it approaches a near-straight line with random drift (near $H=1$ ). This result is significant because it does not stem from the autocovariance properties of the strategies' expected returns; indeed, for any Hurst exponent, the instantaneous forecast is a white noise. Instead, the result reflects the magnitude of the 
Fig. 1 Profits per unit of risk and time (vertical), i.e., the expression in (2.1) divided by $T / \gamma$, against Hurst exponent (horizontal)

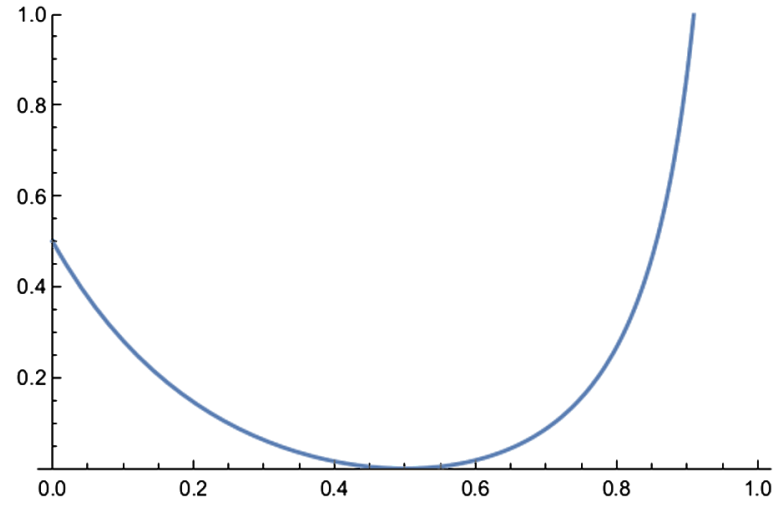

variance of the white-noise forecast that is extracted from the paths of $\mathrm{fBm}$ for different values of $H$ : for $H$ near zero, the weights of the white-noise forecast are small and highly concentrated on recent increments, which results in a moderate variance. By contrast, for $H$ near one, the forecast's weights are large and reach far into the path's past history, leading to a diverging variance.

Third, and contrary to the intuition from previous results, including our own, we find that such performance is immune to small frictions - such as proportional transaction costs or immediate nonlinear price impact (Theorem 3.1 and Corollary 3.2). Specifically, while frictions detract from performance, their effect vanishes arbitrarily quickly by slowly increasing the trading frequency as trading costs decrease, so that their asymptotic impact vanishes at any required rate. Similarly, holding trading costs constant, their effect also vanishes by increasing the horizon while appropriately calibrating the trading frequency.

Fourth, we observe that approximations of the latent drift of fractional Brownian motion converge weakly, but not in norm. This observation highlights a qualitative difference between the familiar drifts of diffusions and their partial analogies for fractional processes. Not only are diffusive drifts of the order of infinitesimal time intervals (informally, $d t$ ) while fractional drifts are a power thereof (informally, $\left.(d t)^{H}\right)$; in addition, diffusive drifts can be understood as close approximations of conditionally expected increments over any sufficiently small interval because such approximations converge (in norm) as random variables. By contrast, fractional drifts are critically dependent on the specific interval: as the interval length declines to zero, the conditionally expected returns converge in law, but not as random variables in any reasonable sense.

Finally, it is worthwhile comparing the findings in this paper to the recent results in Guasoni et al. [9], as both articles study optimal trading strategies for fractional Brownian motion, though in very different settings. The main difference lies in the objective functions considered - here a local mean-variance criterion on a finite interval, while in [9] a risk-neutral target with a long horizon. In particular, the presence of a nonlinear friction is crucial to make the problem in [9] well posed, as it would otherwise lead to unbounded expected profits. In contrast, the present local meanvariance criterion is well posed even without frictions as the instantaneous Sharpe ratio remains bounded for any $H \in(0,1)$, although arbitrage is feasible on any inter- 
val because arbitrage profits remain dispersed. Both [9] and the present paper lead to finite maximal Sharpe ratios that are asymmetric in $H$, but their skews are reversed and arise for different reasons: while the asymptotically optimal strategies in [9] have higher Sharpe ratios near zero than near one, they are not necessarily optimal as the strategies maximise a risk-neutral objective, not the Sharpe ratio. By contrast, the Sharpe ratios obtained here are indeed optimal as they maximise the local meanvariance criterion over any finite interval by ergodicity.

The rest of the paper is organised as follows. Section 2 describes the model and the main result without frictions, discussing their significance and implications. Section 3 considers frictions and shows how their impact can be mitigated by a judicious choice of the trading frequency. Section 4 concludes, and all proofs are in the Appendix.

\section{Main results}

An investor trades a safe and a risky asset. The safe rate is assumed zero to simplify notation, while the price of the risky asset is a multiple of fractional Brownian motion.

Definition 2.1 Fractional Brownian motion $(\mathrm{fBm})$ with Hurst index $H \in(0,1)$ is a Gaussian process $B^{H}=\left(B_{t}^{H}\right)_{t \geq 0}$ defined on a probability space $(\Omega, \mathcal{F}, P)$, with continuous trajectories such that $E\left[B_{t}^{H} B_{s}^{H}\right]=\frac{1}{2}\left(t^{2 H}+s^{2 H}-|t-s|^{2 H}\right), t, s \geq 0$, and $E\left[B_{t}^{H}\right]=0, t \geq 0$.

The case $H=1 / 2$ corresponds to usual Brownian motion, henceforth excluded. Thus $H \in(0,1) \backslash\{1 / 2\}$ unless stated otherwise. Consider a trading horizon $T>0$ and a frequency $n \geq 1$, which represents the number of trading periods in the interval $[0, T]$. The set $\Sigma_{n}$ of strategies consists of sequences $\pi_{s}, s \in\{T k / n: 0 \leq k \leq n-1\}$, of random variables such that $\pi_{s}$ is $\mathcal{F}_{s}$-measurable for all such $s$, where $\left(\mathcal{F}_{S}\right)_{s \geq 0}$ is the augmented natural filtration of $B^{H}$.

An investor who holds at the beginning of each interval $[T k / n, T(k+1) / n]$ a number of shares equal to $\pi_{T k / n}$ attains the mean-variance performance

$$
\begin{aligned}
\operatorname{MV}(\pi, k, n, S):= & E_{T k / n}\left[\pi_{T k / n}\left(S_{T(k+1) / n}-S_{T k / n}\right)\right] \\
& -\frac{\gamma}{2} \operatorname{Var}_{T k / n}\left[\pi_{T k / n}\left(S_{T(k+1) / n}-S_{T k / n}\right)\right],
\end{aligned}
$$

where $E_{t}[X]$ and $\operatorname{Var}_{t}[X]:=E_{t}\left[X^{2}\right]-\left(E_{t}[X]\right)^{2}$ respectively denote the conditional expectation and conditional variance of a random variable $X$ with respect to $\mathcal{F}_{t}$, while $S_{t}=\sigma B_{t}^{H}$ denotes the risky asset price at time $t$. The parameter $\gamma>0$ represents the investor's aversion to risk (as measured by variance).

Assuming time-additive preferences, the overall performance in the interval $[0, T]$ of a trading strategy is defined as the sum of the per-period performances, weighing each period by its length $T / n$, i.e.,

$$
R_{\gamma}(\pi, n, S):=\frac{T}{n} \sum_{k=0}^{n-1} \operatorname{MV}(\pi, k, n, S)=\int_{0}^{T} \operatorname{MV}(\pi,\lfloor t n\rfloor, n, S) d t
$$


Thus the high-frequency objective for fractional Brownian motion is

$$
V(H, \gamma):=\limsup _{n \rightarrow \infty} \sup _{\pi \in \Sigma_{n}} E\left[R_{\gamma}\left(\pi, n, \sigma B^{H}\right)\right]
$$

and represents the maximal performance of a continuous-time strategy that updates the portfolio at arbitrary frequency on the interval $[0, T]$.

With this notation, the main result of this paper is

Theorem 2.2 For each $H \in(0,1)$,

$$
V(H, \gamma)=\frac{T}{\gamma}\left(\frac{\Gamma(H+1 / 2) \Gamma(2-2 H)}{2 \Gamma(3 / 2-H)}-\frac{1}{2}\right),
$$

and the limit superior in the definition of $V(H, \gamma)$ is in fact a limit.

Before discussing the details of this result, it is useful to compare it to the familiar benchmark of Brownian motion with drift, i.e.,

$$
S_{t}=\mu t+\sigma W_{t},
$$

for some Brownian motion $W$ and $\mu \in \mathbb{R}, \sigma>0$. A simple calculation then shows that

$$
\sup _{\pi \in \Sigma_{n}} E\left[R_{\gamma}(\pi, n, S)\right]=E\left[R_{\gamma}\left(\frac{\mu}{\gamma \sigma^{2}}, n, S\right)\right]=\frac{\mu^{2}}{2 \gamma \sigma^{2}} T .
$$

In other words, both the optimal strategy and its performance do not depend on $n$, are inversely proportional to the squared volatility $\sigma^{2}$ and risk aversion $\gamma$, and are respectively linear and quadratic in the drift. In addition, performance is linear in the investment horizon. The linear dependence on the drift and the inverse dependence on the volatility is at the heart of the risk-return tradeoff that arises in random-walk models: as returns are serially independent, their randomness is purely a source of risk, and its reduction is unambiguously beneficial.

The fractional high-frequency performance in (2.1) contains surprising features both in its departures and in its analogies with the usual mean-variance performance (2.2). In contrast to (2.2), the performance in (2.1) is independent of volatility. (In fact, the result is also independent of an additional drift, as observed in Remark A.11. Intuitively, the reason is that for a short time interval, the conditionally expected increment of $\mathrm{fBm}$ is of order $(d t)^{H}$, which makes an ordinary drift of order $d t$ negligible in the mean-variance optimal strategy and its performance.) As shown below, the optimal strategy inversely depends on variance, but this dependence is lost in performance because the expected return directly depends on variance, thereby offsetting its effect.

In analogy to (2.2), the performance in (2.1) is linear in the investment horizon. Upon reflection, also such an analogy is surprising because the linearity in the horizon of the usual mean-variance performance in (2.2) stems from the independence of increments of Brownian motion and the constant drift. Instead, the dependence in increments of fractional Brownian motion is substantial and indeed crucial to generate positive returns. 
The dependence on the Hurst exponent $H$, displayed in Fig. 1, is similarly puzzling in view of its asymmetry. At one extreme, as $H$ approaches zero and increments increasingly resemble white noise (Mishura [15, Lemma 4.1]), performance converges to a finite limit, i.e.,

$$
V(H, \gamma)=\frac{T}{\gamma}\left(\frac{\Gamma(1 / 2) \Gamma(2)}{2 \Gamma(3 / 2)}-\frac{1}{2}\right)+O(H)=\frac{T}{2 \gamma}+O(H) .
$$

(The last equality follows from the identity $\Gamma\left(\frac{3}{2}\right)=\frac{1}{2} \Gamma\left(\frac{1}{2}\right)$.) As $H$ approaches $1 / 2$, performance flattens around zero as the process mimics an ordinary Brownian motion. This expansion exploits identities involving the derivatives of the Gamma function (cf. Sun and Qin [21]), namely

$$
V(H, \gamma)=\frac{\pi^{2}}{6}(H-1 / 2)^{2} \frac{T}{\gamma}+O\left((H-1 / 2)^{3}\right) .
$$

In particular, this identity confirms the intuition from Fig. 1 that performance reaches its unique minimum of zero in the martingale case of $H=1 / 2$, while slowly increasing in each direction. At the other extreme, as $H$ approaches one and the process resembles a straight line with random slope, performance diverges, i.e.,

$$
V(H, \gamma)=\left(\frac{1}{8(1-H)}+\frac{-3+\log 4}{4}\right) \frac{T}{\gamma}+O(1-H) .
$$

To obtain the term $1 /(8(1-H))$, recall that $\Gamma(x) \sim 1 / x$ for $x$ near zero. The term $(-3+\log 4) / 4$ follows from more complex higher-order asymptotics. Key to understanding these features is the prediction mechanism at the heart of the problem. As our mean-variance objective is time-additive, the optimal trading strategies maximise performance in the next period. Because for a square-integrable random variable $X$, the functional $\varphi \mapsto E[\varphi X]-\frac{\gamma}{2} \operatorname{Var}[\varphi X]$ attains its maximum $E^{2}[X] /(2 \gamma \operatorname{Var}[X])$ at $\phi^{*}=E[X] /(\gamma \operatorname{Var}[X])$, the optimal strategy $\pi(n) \in \Sigma_{n}$ is

$$
\pi_{T k / n}(n):=\frac{E_{T k / n}\left[B_{T(k+1) / n}^{H}-B_{T k / n}^{H}\right]}{\gamma \operatorname{Var}_{T k / n}\left[B_{T(k+1) / n}^{H}-B_{T k / n}^{H}\right]}, \quad 0 \leq k \leq n-1 .
$$

To investigate the high-frequency limit, it is convenient to extend these strategies by right-continuity to the entire interval $[0, T]$, i.e., setting

$$
\pi_{t}(n):=\pi_{T k / n}(n), \quad t \in[T k / n, T(k+1) / n), 0 \leq k<n .
$$

With this notation, the next theorem identifies the limit of such strategies, which is interpreted as the asymptotically optimal strategy in the high-frequency regime.

Theorem 2.3 The sequence $\left(\left((T / n)^{-H} \pi_{t}(n)\right)_{t \in[0, T]}\right)_{n \in \mathbb{N}}$ consists of Gaussian processes that, as $n$ increases, converge in finite-dimensional distributions to a Gaussian process $\left(B_{t}\right)_{t \in[0, T]}$ such that

1) $B_{0}=0$ a.s.

2) $E\left[B_{t}\right]=0, t \in(0, T]$. 
Fig. 2 Autocorrelation (vertical axis) of the strategies $\pi_{T k / n}(n)$ (equivalently, of the expected increments) against time lag (horizontal) as the frequency $n$ increases from 100 (top) to 200 , 500 and 1000 (bottom). The autocorrelation converges to the white-noise limit of one at lag zero and zero elsewhere. Each plotted curve is the average of 1000 sample autocorrelograms with $H=0.6$

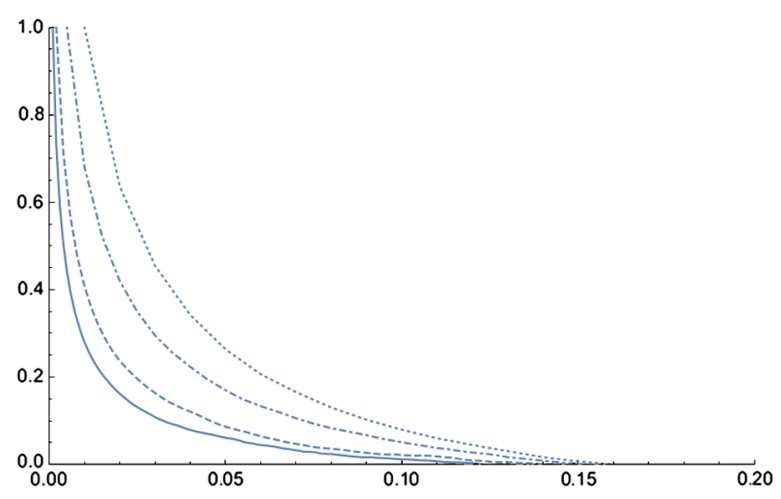

3) $E\left[B_{t}^{2}\right]=1-\frac{\Gamma(3 / 2-H)}{\Gamma(H+1 / 2) \Gamma(2-2 H)}, t \in(0, T]$.

4) $E\left[B_{t} B_{s}\right]=0$ for $t \neq s, s, t \in[0, T]$.

Proof Follows from Proposition A.5, Lemma A.7 and Theorem A.8 below.

This result has a striking message: Up to a scaling factor, the optimal strategy hence the expected return over the next period - is essentially a white noise (the exception is $t=0$, for which the process is conventionally pinned at zero). In other words, regardless of the Hurst exponent $H$ and regardless of the autocorrelation of increments in fractional Brownian motion, the forecasts of short-term increments (i.e., the trading signals) are virtually uncorrelated from one instant to the next. Figure 2 illustrates the convergence result in the theorem by plotting at increasing frequencies the autocorrelation of $\pi_{T k / n}(n)$, which converges to the autocorrelation of a white noise.

The Hurst exponent controls the scale of the strategy: Denoting by $\Delta$ the length of each trading period, price increments have conditional expectation of order $\Delta^{H}$ and conditional variance of order $\Delta^{2 H}$, which implies trading positions of order $\Delta^{-H}$ (cf. Proposition A.5 and Theorem A.8). This feature is in contrast to the Brownian benchmark, in which both the expected return $\mu \Delta$ and its variance $\sigma^{2} \Delta$ are of the same order. Instead, the variance in the fractional setting has a smaller order, which means that bets become more favourable as the trading frequency increases, and therefore their optimal size increases.

Note, however, that the implied performance in each period is proportional to the conditional expectation $\Delta^{H}$ times the position size $\Delta^{-H}$, hence of order 1 . As each trading period leads to the same performance (in view of the white-noise property established in Theorem 2.3), trading over an interval of length $T$ generates a performance proportional to $T$. In particular, the results below show that the optimal trading position is asymptotically

$$
\varphi_{t}(\Delta):=\frac{\Delta^{-H}}{\gamma} \frac{\Gamma(H+1 / 2) \Gamma(2-2 H)}{\Gamma(3 / 2-H)} B_{t},
$$


and that on the subsequent interval, the expected increment has the asymptotic (conditional) mean and variance

$$
m_{t}(\Delta):=B_{t} \Delta^{H}, \quad v(\Delta):=\frac{\Gamma(3 / 2-H)}{\Gamma(H+1 / 2) \Gamma(2-2 H)} \Delta^{2 H} .
$$

The performance formula in Theorem 2.2 follows from

$$
\begin{aligned}
T E\left[\varphi_{t}(\Delta) m_{t}(\Delta)-\frac{\gamma}{2} \varphi_{t}(\Delta)^{2} v(\Delta)\right] & =\frac{T}{2 \gamma} \frac{\Gamma(H+1 / 2) \Gamma(2-2 H)}{\Gamma(3 / 2-H)} E\left[B_{t}^{2}\right] \\
& =\frac{T}{\gamma}\left(\frac{\Gamma(H+1 / 2) \Gamma(2-2 H)}{2 \Gamma(3 / 2-H)}-\frac{1}{2}\right) .
\end{aligned}
$$

This analysis also offers an intuitive explanation for the asymmetric behaviour of the performance in (2.3) and (2.4). For $H$ close to zero, the asset price $S$ itself is akin to a white noise, for which the mean and variance in (2.6) are of the same order. Accordingly, the performance converges to a finite limit. By contrast, for $H$ close to one, the process degenerates to a straight line with random slope, as randomness vanishes from its increments. Thus the trading strategy generates return with virtually no risk, and the performance diverges.

Note that the mean-variance optimal strategies $\left(\pi_{t}(n)\right)$ are not arbitrage opportunities as the support of their payoffs is $(-\infty,+\infty)$. Although continuous trading with fBm leads to arbitrage opportunities (see e.g. Rogers [19], Salopek [20]), it is clear that on any finite deterministic grid, fBm does not admit arbitrage because an equivalent martingale measure can be constructed through a backward recursion that aligns all conditionally expected increments to zero. (In fact, arbitrage disappears even when a minimal time has to pass between two subsequent transactions; see Cheridito [2].)

A deeper question is whether the sequence of strategies $(\pi(n))_{n \geq 1}$ yields an arbitrage in some limit sense, and the answer is affirmative. The sequence of discrete-time mean-variance optimal policies offers a statistical arbitrage in that

$$
\lim _{n \rightarrow \infty} E[W(n)]=\infty,
$$

where $W(n)$ is the final wealth of the strategy $\pi(n)$ starting from null initial capital, i.e.,

$$
W(n)=\sum_{k=0}^{n-1} \pi_{T k / n}(n)\left(S_{T(k+1) / n}-S_{T k / n}\right) .
$$

This fact is readily proved by observing that in mean-variance optimisation, the expectation of the optimal strategy is always twice as large as its variance, whence

$$
\lim _{n \rightarrow \infty} E[W(n)]=\lim _{n \rightarrow \infty} \frac{2 n}{T} E\left[R_{\gamma}(\pi(n), n, S)\right]=\infty,
$$

because $E\left[R_{\gamma}(\pi(n), n, S)\right]$ tends to a finite nonzero limit (for $H \neq 1 / 2$ ) by Theorem 2.2 .

As Theorem 2.3 establishes that the rescaled strategies essentially converge to a white noise in finite-dimensional distributions, a natural question is whether such a 
convergence holds in a stronger sense, such as in square norm, so that its limit can be interpreted as a rescaled asymptotically optimal strategy in continuous time.

The next result provides a negative answer to this question by showing that even focusing on a sequence of dyadic partitions, the square norm between each discretisation and the next remains bounded away from zero.

Theorem 2.4 Let $\Delta_{k}=T / k$. For all $H \in(0,1) \backslash\{1 / 2\}$ and $t \in(0, T)$,

$$
\lim _{n \rightarrow \infty} E\left[\left(\Delta_{2^{n}}^{-H} \pi_{t}\left(2^{n}\right)-\Delta_{2^{n+1}}^{-H} \pi_{t}\left(2^{n+1}\right)\right)^{2}\right]>0 .
$$

The significance of this result is that the optimal strategy is extremely sensitive to the trading frequency used, and that optimal strategies at increasing frequencies are not approximations of some underlying continuous-time strategy, which does not exist. In fact, even if such a strategy existed, it would be of no use because the paths of a white-noise process are not even measurable (cf. Revuz and Yor [18, p. 37]).

At a more concrete level, the above results show that as the frequency increases, the corresponding trading strategies become increasingly variable; thus in practice, their ostensible theoretical performance may be more than offset by the trading costs that such strategies entail. The next section investigates this issue by identifying how the optimal trading frequency depends on the size of trading costs.

\section{Trading costs}

The optimal strategies identified in (2.5) imply that asset positions are both large and highly variable, thereby calling into question their robustness to trading costs. To investigate this issue, recall the sequence of strategies $\pi(n), n \geq 1$, defined in (2.5) above.

Assuming that a portfolio change from $\theta_{1}$ to $\theta_{2}$ shares incurs the cost $\lambda\left|\theta_{1}-\theta_{2}\right|^{\alpha}$ for some $\alpha, \lambda>0$, the local mean-variance analysis for a trader applying the strategy $\pi(n)$ leads to the functionals (setting $\pi_{t}(n):=0$ for $t<0$ )

$$
\tilde{R}(n):=R(n)-\sum_{k=0}^{n-1} \lambda\left|\pi_{T k / n}(n)-\pi_{T(k-1) / n}(n)\right|^{\alpha},
$$

where $R(n)$ denotes frictionless performance, i.e.,

$$
\begin{aligned}
R(n):= & \frac{T}{n} \sum_{k=0}^{n-1} E_{T k / n}\left[\pi_{T k / n}(n)\left(S_{T(k+1) / n}-S_{T k / n}\right)\right] \\
& -\frac{\gamma}{2} \frac{T}{n} \sum_{k=0}^{n-1} \operatorname{Var}_{T k / n}\left[\pi_{T k / n}(n)\left(S_{T(k+1) / n}-S_{T k / n}\right)\right],
\end{aligned}
$$

while the second term in $\tilde{R}(n)$ represents the effect of trading costs. The next result shows that expected trading costs $E[R(n)-\tilde{R}(n)]$ grow with a superlinear power of 
the trading frequency $n$ that increases with both the Hurst and the friction exponents. As a result, for fixed transaction costs, the objective function arbitrarily deteriorates as the frequency increases, and the optimal trading frequency must be finite.

Theorem 3.1 $E[R(n)-\tilde{R}(n)]=O\left(n^{1+\alpha H}\right)$ and hence $\lim _{n \rightarrow \infty} E[\tilde{R}(n)]=-\infty$.

The next logical step is to understand the effect of small trading costs on the overall objective. Here the above result leads to an unexpected implication: with a judicious choice of the trading frequency, the effect of frictions is negligible at any order.

Corollary 3.2 Let $n_{\lambda}=\left\lfloor\lambda^{\frac{\beta-1}{1+\alpha H}}\right\rfloor$ for $\beta \in(0,1)$. Then

$$
E\left[R\left(n_{\lambda}\right)-\tilde{R}\left(n_{\lambda}\right)\right]=O\left(\lambda^{\beta}\right) \quad \text { for } \lambda \rightarrow 0,
$$

that is, trading costs are of order $\lambda^{\beta}$.

Upon reflection, this result is a direct consequence of Theorem 3.1. Yet, its conclusion is counterintuitive when compared to the results for frictions in familiar diffusion models (cf. Guasoni and Weber [11, Theorem 4.1]) where the welfare loss is of the order of $\lambda^{\frac{2}{2+\alpha}}$; for example, proportional transaction costs correspond to $\alpha=1$, leading to a welfare loss of order $2 / 3$.

Intuitively, the main difference is that in familiar diffusion models, the main determinant of optimal portfolios is the asset price's local drift which is typically smooth. Thus as the trading frequency increases, smaller and smaller adjustments are required, which means that holding trading costs constant, the high-frequency limit of the portfolio performance is finite.

In contrast, in the fractional setting considered here, the "latent drift" of the process is highly irregular - in the limit, it is a white noise -; hence it entails trading costs that grow with the trading frequency as implied by Theorem 3.1. However, this irregularity can be harnessed to make trading costs negligible in the high-frequency limit, by choosing the trading frequency $n_{\lambda}$ to grow slowly as $\lambda$ decreases so that overall costs vanish in the limit. Of course, letting $n$ grow more slowly has the downside that the convergence of the strategy's performance to the optimum in (2.1) is also going to be slower.

Corollary 3.2 also identifies the maximal speed identifies the maximal speed at which the frequency may grow so that the strategy converges to the optimum. In particular, $n_{\lambda}$ may grow at a rate arbitrarily close to $\lambda^{-\frac{1}{1+\alpha H}}$, but not at this exact rate: at this critical regime, costs would not vanish but converge to a positive finite limit, which would be suboptimal.

As trading costs are fixed in applications, the significance of this result is as follows: In practice, the trading cost $\lambda$ implies that the optimal trading interval should be $1 / n_{\lambda} \approx \lambda^{\frac{1-\beta}{1+\alpha H}}$, where $\beta$ is close to one. However, the closer the $\beta$ to one, the larger the trading interval, which means that the convergence of the strategy to the frictionless limit for a fixed horizon $T$ is slower, and its risk higher. Thus if the horizon is not long enough to guarantee that the payoff has a sufficiently low risk, one may choose to decrease the value of $\beta$ to reduce risk further, at the price of an increased trading cost. 


\section{Conclusion}

This paper finds locally mean-variance optimal trading strategies for an asset price that follows fractional Brownian motion, and finds that the average Sharpe ratio is finite, asymmetric in the Hurst exponent, bounded near zero, and unbounded near one. The central result is that conditionally expected increments are asymptotically a Gaussian white noise, regardless of the Hurst exponent, but with a variance that depends on that exponent.

The optimal performance is insensitive to small trading frictions, in that their impact can be mitigated arbitrarily well by calibrating the trading frequency appropriately. This phenomenon is in sharp contrast to diffusion models for which the impact of small frictions has a fixed order of magnitude.

Acknowledgements We are grateful to the Co-Editor Masaaki Fukasawa, the Associate Editor and two anonymous referees for their perceptive and insightful comments, which helped improve the paper significantly.

Funding Note Open access funding provided by ELKH Alfréd Rényi Institute of Mathematics.

Publisher's Note Springer Nature remains neutral with regard to jurisdictional claims in published maps and institutional affiliations.

Open Access This article is licensed under a Creative Commons Attribution 4.0 International License, which permits use, sharing, adaptation, distribution and reproduction in any medium or format, as long as you give appropriate credit to the original author(s) and the source, provide a link to the Creative Commons licence, and indicate if changes were made. The images or other third party material in this article are included in the article's Creative Commons licence, unless indicated otherwise in a credit line to the material. If material is not included in the article's Creative Commons licence and your intended use is not permitted by statutory regulation or exceeds the permitted use, you will need to obtain permission directly from the copyright holder. To view a copy of this licence, visit http://creativecommons.org/licenses/by/4.0/.

\section{Appendix: Proofs}

Henceforth, the expectation $E[X]$ of a random variable $X$ is defined as $-\infty$ when both $E\left[X^{+}\right], E\left[X^{-}\right]$are infinite. Recall also the notion of asymptotic equivalence, where $f(x) \sim g(x)$ near $x=x_{0}$ means that $\lim _{x \rightarrow x_{0}} f(x) / g(x)=1$.

\section{A.1 Auxiliary results on fractional Brownian motion}

For $s, t \geq 0$, introduce the kernel

$$
\begin{aligned}
Z_{H}(t, s):=c_{H} & \left(t^{H-\frac{1}{2}} s^{\frac{1}{2}-H}(t-s)^{H-\frac{1}{2}}\right. \\
& \left.-\left(H-\frac{1}{2}\right) s^{\frac{1}{2}-H} \int_{s}^{t} u^{H-\frac{3}{2}}(u-s)^{H-\frac{1}{2}} d u\right) 1_{\{0<s<t\}},
\end{aligned}
$$

where

$$
c_{H}=\left(\frac{2 H \Gamma(3 / 2-H)}{\Gamma(H+1 / 2) \Gamma(2-2 H)}\right)^{1 / 2} .
$$


Then, taking an (ordinary) Brownian motion $W=\left(W_{t}\right)_{t \geq 0}$, the formula

$$
B_{t}^{H}:=\int_{0}^{t} Z_{H}(t, s) d W_{s}, \quad t \geq 0,
$$

defines an $\mathrm{fBm}$ with parameter $H$ which generates the same filtration as $W$ (cf. [16, Theorem 3.2]). Moreover, any fractional Brownian motion allows the representation (A.2) with some Wiener process $W$, and both processes generate the same filtration. Fixing such a representation, denote $\mathcal{F}_{t}:=\sigma\left(W_{s}, 0 \leq s \leq t\right)$.

The kernel representation in (A.2) implies the following properties of fractional increments.

Proposition A.1 For any $0 \leq z \leq u$,

$$
\begin{aligned}
B_{u}^{H}-B_{z}^{H} & =\int_{0}^{z}\left(Z_{H}(u, s)-Z_{H}(z, s)\right) d W_{s}+\int_{z}^{u} Z_{H}(u, s) d W_{s}, \\
E_{z}\left[B_{u}^{H}-B_{z}^{H}\right] & =\int_{0}^{z}\left(Z_{H}(u, s)-Z_{H}(z, s)\right) d W_{s}, \\
E_{z}\left[\left(B_{u}^{H}-B_{z}^{H}\right)^{2}\right] & =\left(\int_{0}^{z}\left(Z_{H}(u, s)-Z_{H}(z, s)\right) d W_{s}\right)^{2}+\int_{z}^{u} Z_{H}^{2}(u, s) d s .
\end{aligned}
$$

Proof The relations immediately follow from (A.2).

In particular, note that for $z=0$,

$$
E_{z}\left[B_{u}^{H}-B_{z}^{H}\right]=E\left[B_{u}^{H}-B_{z}^{H}\right]=0 .
$$

As all the relations (A.3)-(A.5) contain the increment $Z_{H}(u, s)-Z_{H}(z, s)$, it is useful to rewrite this expression in a more convenient form.

Lemma A.2 Let $0 \leq s<z<u$. Then

$$
Z_{H}(u, s)-Z_{H}(z, s)=(H-1 / 2) c_{H} s^{1 / 2-H} \int_{z}^{u} v^{H-1 / 2}(v-s)^{H-3 / 2} d v .
$$

Proof For $H>1 / 2$, (A.6) follows by integrating (A.1) by parts. For $H<1 / 2$, (A.2) implies that

$$
\begin{aligned}
& Z_{H}(u, s)-Z_{H}(z, s) \\
& =c_{H} s^{1 / 2-H}\left(u^{H-1 / 2}(u-s)^{H-1 / 2}-(H-1 / 2) \int_{z}^{u} v^{H-3 / 2}(v-s)^{H-1 / 2} d v\right. \\
& \left.\quad-z^{H-1 / 2}(z-s)^{H-1 / 2}\right) .
\end{aligned}
$$

Integrating $\int_{z}^{u} v^{H-3 / 2}(v-s)^{H-1 / 2} d u$ by parts gives

$$
\begin{aligned}
& \int_{z}^{u} v^{H-3 / 2}(v-s)^{H-1 / 2} d v \\
& =\frac{u^{H-1 / 2}(u-s)^{H-1 / 2}}{H-1 / 2}-\frac{z^{H-1 / 2}(z-s)^{H-1 / 2}}{H-1 / 2}-\int_{z}^{u} v^{H-1 / 2}(v-s)^{H-3 / 2} d v .
\end{aligned}
$$


Note that this expression can be integrated by parts, while the kernel $Z_{H}(u, s)$ itself cannot, because the integral $\int_{z}^{u} v^{H-1 / 2}(v-s)^{H-3 / 2} d v$ exists for $z>s$, but diverges for $z=s$. Therefore, for $0 \leq s<z$ and $0<H<1 / 2$, (A.7) implies that

$$
Z_{H}(u, s)-Z_{H}(z, s)=c_{H}(H-1 / 2) s^{1 / 2-H} \int_{z}^{u} v^{H-1 / 2}(v-s)^{H-3 / 2} d v,
$$

which coincides with (A.6).

Let $\tilde{c}_{H}:=c_{H}(H-1 / 2)$. In view of (A.6), equations (A.3)-(A.5) admit the following alternative representation.

Proposition A.3 For $z<u$, denote

$$
\xi_{u, z}:=\int_{0}^{z} s^{1 / 2-H}\left(\int_{z}^{u} v^{H-1 / 2}(v-s)^{H-3 / 2} d v\right) d W_{s} .
$$

For any $H \in(0,1) \backslash\{1 / 2\}$, we have

$$
\begin{aligned}
B_{u}^{H}-B_{z}^{H} & =\tilde{c}_{H}\left(\xi_{u, z}+\int_{z}^{u} Z_{H}(u, s) d W_{s}\right), \\
E_{z}\left[B_{u}^{H}-B_{z}^{H}\right] & =\tilde{c}_{H} \xi_{u, z}, \\
E_{z}\left[\left(B_{u}^{H}-B_{z}^{H}\right)^{2}\right] & =\left(\tilde{c}_{H} \xi_{u, z}\right)^{2}+\int_{z}^{u} Z_{H}^{2}(u, s) d s, \\
\operatorname{Var}_{z}\left[B_{u}^{H}-B_{z}^{H}\right] & =\int_{z}^{u} Z_{H}^{2}(u, s) d s .
\end{aligned}
$$

Proof Follows from Lemma A.2 and Proposition A.1.

\section{A.2 Variance bounds for conditionally expected increments}

Henceforth, assume that $z>0$. Recall that $\xi_{u, z}$ is a centered Gaussian random variable with variance

$$
E\left[\xi_{u, z}^{2}\right]=\int_{0}^{z} s^{1-2 H}\left(\int_{z}^{u} v^{H-1 / 2}(v-s)^{H-3 / 2} d v\right)^{2} d s .
$$

The goal is to find lower and upper bounds for the right-hand side. Thus set

$$
\begin{aligned}
J_{u, z} & :=(H-1 / 2)^{-2} \int_{0}^{\frac{z}{2(u-z)}}\left(1-\frac{u-z}{z} r\right)^{1-2 H}\left((1+r)^{H-1 / 2}-r^{H-1 / 2}\right)^{2} d r, \\
I_{u, z}^{b} & :=z^{2 H-2}(u-z)^{2} \int_{0}^{1 / 2} s^{1-2 H}\left(\frac{u}{z}-s\right)^{2 H-3} d s+(u-z)^{2 H} J_{u, z}, \\
\widetilde{I}_{u, z}^{\mathrm{b}} & :=\frac{u^{2 H-1}}{z}(u-z)^{2} \int_{0}^{1 / 2} s^{1-2 H}\left(\frac{u}{z}-s\right)^{2 H-3} d s+(u-z)^{2 H} J_{u, z}, \\
I_{u, z}^{\sharp} & :=\frac{u^{2 H-1}}{z}(u-z)^{2} \int_{0}^{1 / 2} s^{1-2 H}(1-s)^{2 H-3} d s+(u / z)^{2 H-1}(u-z)^{2 H} J_{u, z} .
\end{aligned}
$$


Lemma A.4 For $H>1 / 2$,

$$
\tilde{c}_{H}^{2} I_{u, z}^{\mathrm{b}} \leq \tilde{c}_{H}^{2} E\left[\xi_{u, z}^{2}\right]=E\left[E_{z}^{2}\left[B_{u}^{H}-B_{z}^{H}\right]\right] \leq \tilde{c}_{H}^{2} I_{u, z}^{\sharp},
$$

and for $H<1 / 2$,

$$
\tilde{c}_{H}^{2} \widetilde{I}_{u, z}^{\mathrm{b}} \leq \tilde{c}_{H}^{2} E\left[\xi_{u, z}^{2}\right]=E\left[E_{z}^{2}\left[B_{u}^{H}-B_{z}^{H}\right]\right] \leq \tilde{c}_{H}^{2} \widetilde{I}_{u, z}^{\sharp} .
$$

Proof (i) Let $H>1 / 2$. Consider the integral

$$
\begin{aligned}
I_{u, z}^{0} & :=\int_{0}^{z} s^{1-2 H}\left(\int_{z}^{u}(v-s)^{H-3 / 2} d v\right)^{2} d s \\
& =(H-1 / 2)^{-2} \int_{0}^{z} s^{1-2 H}\left((u-s)^{H-1 / 2}-(z-s)^{H-1 / 2}\right)^{2} d s .
\end{aligned}
$$

Then obviously

$$
z^{2 H-1} I_{u, z}^{0} \leq E\left[\xi_{u, z}^{2}\right] \leq u^{2 H-1} I_{u, z}^{0} .
$$

To estimate $I_{u, z}^{0}$ from below and from above, the change of variables $s=z x$ gives

$$
\begin{aligned}
I_{u, z}^{0}= & (H-1 / 2)^{-2} z \int_{0}^{1} x^{1-2 H}\left((u / z-x)^{H-1 / 2}-(1-x)^{H-1 / 2}\right)^{2} d x \\
= & (H-1 / 2)^{-2} z\left(\int_{0}^{1 / 2} x^{1-2 H}\left((u / z-x)^{H-1 / 2}-(1-x)^{H-1 / 2}\right)^{2} d x\right. \\
& \left.\quad+\int_{1 / 2}^{1} x^{1-2 H}\left((u / z-x)^{H-1 / 2}-(1-x)^{H-1 / 2}\right)^{2} d x\right) \\
= & (H-1 / 2)^{-2} z\left(I_{u, z}^{1}+I_{u, z}^{2}\right) .
\end{aligned}
$$

To estimate $I_{u, z}^{1}$, note that the Lagrange mean value theorem implies

$$
\left(\frac{u}{z}-s\right)^{H-1 / 2}-(1-s)^{H-1 / 2}=\left(\frac{u}{z}-1\right)(H-1 / 2)(\theta-s)^{H-3 / 2}
$$

for some $\theta \in(1, u / z)$. Therefore

$$
\begin{aligned}
& \left(\frac{u-z}{z}\right)^{2}(H-1 / 2)^{2} \int_{0}^{1 / 2} s^{1-2 H}\left(\frac{u}{z}-s\right)^{2 H-3} d s \\
& \leq I_{u, z}^{1} \\
& \leq\left(\frac{u-z}{z}\right)^{2}(H-1 / 2)^{2} \int_{0}^{1 / 2} s^{1-2 H}(1-s)^{2 H-3} d s .
\end{aligned}
$$


Turning to $I_{u, z}^{2}$, the changes of variables $1-x=y$ and $y=\frac{u-z}{z} r$ yield

$$
\begin{aligned}
& I_{u, z}^{2} \\
& =\int_{0}^{1 / 2}(1-y)^{1-2 H}\left(\left(\frac{u-z}{z}+y\right)^{H-1 / 2}-y^{H-1 / 2}\right)^{2} d y \\
& =\left(\frac{u-z}{z}\right)^{2 H} \int_{0}^{\frac{z}{2(u-z)}}\left(1-\frac{u-z}{z} r\right)^{1-2 H}\left((1+r)^{H-1 / 2}-r^{H-1 / 2}\right)^{2} d r .
\end{aligned}
$$

Finally, from (A.9)-(A.11) and (A.8), it follows that

$$
I_{u, z}^{b} \leq E\left[\xi_{u, z}^{2}\right] \leq I_{u, z}^{\sharp} .
$$

(ii) Let $H<1 / 2$. Note that in this case,

$$
u^{2 H-1} I_{u, z}^{0} \leq E\left[\xi_{u, z}^{2}\right] \leq z^{2 H-1} I_{u, z}^{0}
$$

Equality (A.9) holds true. For $I_{u, z}^{1}$, we have (A.10), and (A.11) also holds true. Substituting (A.9)-(A.11) into (A.12), it now follows that the lower and upper bounds equal $\tilde{c}_{H}^{2} \widetilde{I}_{u, z}^{D}$ and $\tilde{c}_{H}^{2} \widetilde{I}_{u, z}^{\sharp}$, respectively, and the proof is complete.

\section{A.3 Limit variance of the strategies}

Now fix the interval $[0, T]$ and consider the sequence of partitions

$$
\Pi_{n}=\{T k / n, 0 \leq k \leq n\}, \quad n \geq 1,
$$

with mesh $\Delta_{n}:=T / n$. For any point $t \in[0, T]$, denote $\kappa_{t}^{n}:=\left\lfloor\frac{n t}{T}\right\rfloor$, whence

$$
T \kappa_{t}^{n} / n \leq t<T\left(\kappa_{t}^{n}+1\right) / n
$$

Now consider the step functions

$$
\begin{aligned}
\zeta_{t}^{n} & :=\sum_{k=0}^{n-1} E_{T k / n}\left[B_{T(k+1) / n}^{H}-B_{T k / n}^{H}\right] 1_{\{t \in[T k / n, T(k+1) / n)\}} \\
& =\sum_{k=0}^{n-1} \tilde{c}_{H} \xi_{T(k+1) / n, T k / n} 1_{\{t \in[T k / n, T(k+1) / n)\}}=\tilde{c}_{H} \xi_{T\left(\kappa_{t}^{n}+1\right) / n, T \kappa_{t}^{n} / n} .
\end{aligned}
$$

Applying Lemma A.4 with $u:=T\left(\kappa_{t}^{n}+1\right) / n, z=T \kappa_{t}^{n} / n$, lower and upper bounds for $E\left[\left(\zeta_{t}^{n}\right)^{2}\right], t>0$ immediately follow. Namely, assume that $n$ is sufficiently large 
so that $\kappa_{t}^{n}>0$. Setting

$$
\tilde{J}:=(H-1 / 2)^{-2} \int_{0}^{\kappa_{t}^{n} / 2}\left(1-\frac{y}{\kappa_{t}^{n}}\right)^{1-2 H}\left((y+1)^{H-1 / 2}-y^{H-1 / 2}\right)^{2} d y,
$$

note that

$$
\begin{aligned}
I_{T\left(\kappa_{t}^{n}+1\right) / n, T \kappa_{t}^{n} / n}^{\mathrm{b}}= & \left(T \kappa_{t}^{n} / n\right)^{2 H-2} \Delta_{n}^{2} \int_{0}^{1 / 2} s^{1-2 H}\left(\frac{\kappa_{t}^{n}+1}{\kappa_{t}^{n}}-s\right)^{2 H-3} d s+\Delta_{n}^{2 H} \tilde{J}, \\
I_{T\left(\kappa_{t}^{n}+1\right) / n, T \kappa_{t}^{n} / n}^{\sharp}= & \frac{\left(T \frac{\left(\kappa_{t}^{n}+1\right)}{n}\right)^{2 H-1} \Delta_{n}^{2}}{T \frac{\kappa_{t}^{n}}{n}} \int_{0}^{1 / 2} s^{1-2 H}(1-s)^{2 H-3} d s \\
& +\Delta_{n}^{2 H}\left(\frac{\kappa_{t}^{n}+1}{\kappa_{t}^{n}}\right)^{2 H-1} \tilde{J}, \\
\widetilde{I}_{T\left(\kappa_{t}^{n}+1\right) / n, T \kappa_{t}^{n} / n}= & \frac{\left(T \frac{\left(\kappa_{t}^{n}+1\right)}{n}\right)^{2 H-1} \Delta_{n}^{2}}{T \frac{\kappa_{t}^{n}}{n}} \int_{0}^{1 / 2} s^{1-2 H}\left(\frac{\kappa_{t}^{n}+1}{\kappa_{t}^{n}}-s\right)^{2 H-3} d s \\
& +\Delta_{n}^{2 H}\left(\frac{\kappa_{t}^{n}+1}{\kappa_{t}^{n}}\right)^{2 H-1} \tilde{J}, \\
\widetilde{I}_{T\left(\kappa_{t}^{n}+1\right) / n, T \kappa_{t}^{n} / n}^{\sharp} & \left(T \frac{\kappa_{t}^{n}}{n}\right)^{2 H-2} \Delta_{n}^{2} \int_{0}^{1 / 2} s^{1-2 H}(1-s)^{2 H-3} d s+\Delta_{n}^{2 H} \tilde{J} .
\end{aligned}
$$

Using these expressions, the following formula for the limit of variance follows.

Proposition A.5 For any $H \in(0,1) \backslash\{1 / 2\}$,

$$
\begin{aligned}
C_{H}=\lim _{n \rightarrow \infty} \Delta_{n}^{-2 H} E\left[\left(\zeta_{t}^{n}\right)^{2}\right] & =c_{H}^{2} \int_{0}^{\infty}\left((y+1)^{H-1 / 2}-y^{H-1 / 2}\right)^{2} d y \\
& =1-\frac{\Gamma(3 / 2-H)}{\Gamma(H+1 / 2) \Gamma(2-2 H)} .
\end{aligned}
$$

Proof Let $n \rightarrow \infty$. Then $\kappa_{t}^{n} \rightarrow \infty, T \kappa_{t}^{n} / n \uparrow t,\left(\kappa_{t}^{n}+1\right) / \kappa_{t}^{n} \rightarrow 1$. Moreover, Lebesgue's dominated convergence theorem guarantees that

$$
\int_{0}^{1 / 2} s^{1-2 H}\left(\frac{\kappa_{t}^{n}+1}{\kappa_{t}^{n}}-s\right)^{2 H-3} d s \longrightarrow \int_{0}^{1 / 2} s^{1-2 H}(1-s)^{2 H-3} d s .
$$

Consider

$$
\int_{0}^{\kappa_{t}^{n} / 2}\left(1-\frac{y}{\kappa_{t}^{n}}\right)^{1-2 H}\left((y+1)^{H-1 / 2}-y^{H-1 / 2}\right) d y
$$


Note that $1-y / \kappa_{t}^{n} \rightarrow 1$ and it does not exceed 1 for $H<1 / 2$ and $2^{2 H-1}$ for $H>1 / 2$. Therefore Lebesgue's dominated convergence theorem yields

$$
\begin{aligned}
& \lim _{n \rightarrow \infty} \int_{0}^{\kappa_{t}^{n} / 2}\left(1-y / \kappa_{t}^{n}\right)^{1-2 H}\left((y+1)^{H-1 / 2}-y^{H-1 / 2}\right)^{2} d y \\
& =\int_{0}^{\infty}\left((y+1)^{H-1 / 2}-y^{H-1 / 2}\right)^{2} d y .
\end{aligned}
$$

The latter integral is well defined at 0 , and $\left((y+1)^{H-1 / 2}-y^{H-1 / 2}\right)^{2} \sim O\left(y^{2 H-3}\right)$ when $y \rightarrow \infty$; therefore it is also well defined at $\infty$. Furthermore, the first terms in all values

$$
I_{T\left(\kappa_{t}^{n}+1\right) / n, T \kappa_{t}^{n} / n}^{d} \quad \text { and } \quad \widetilde{I}_{T\left(\kappa_{t}^{n}+1\right) / n, T \kappa_{t}^{n} / n}^{d}, \quad d=b, \sharp,
$$

are of order $\Delta_{n}^{2}$, and so they tend to zero when divided by $\Delta_{n}^{2 H}$. The expression on the right-hand side of (A.15) simplifies (cf. Mishura [15, Theorem 1.3.1]) to

$$
\int_{0}^{\infty}\left((y+1)^{H-1 / 2}-y^{H-1 / 2}\right)^{2} d y=\frac{(\Gamma(H+1 / 2))^{2}}{2 H \sin (\pi H) \Gamma(2 H)}-\frac{1}{2 H},
$$

and

$$
c_{H}^{2}=\frac{2 H \Gamma(3 / 2-H)}{\Gamma(H+1 / 2) \Gamma(2-2 H)}
$$

so that

$$
\begin{aligned}
C_{H}: & =c_{H}^{2} \int_{0}^{\infty}\left((y+1)^{H-1 / 2}-y^{H-1 / 2}\right)^{2} d y \\
& =\frac{\Gamma(3 / 2-H) \Gamma(H+1 / 2)}{\sin (\pi H) \Gamma(2 H) \Gamma(2-2 H)}-\frac{\Gamma(3 / 2-H)}{\Gamma(H+1 / 2) \Gamma(2-2 H)} .
\end{aligned}
$$

Now the statement follows from Lemma A.6 below.

Lemma A.6 For any $H \in(0,1) \backslash\{1 / 2\}$, we have the equality

$$
\frac{\Gamma(3 / 2-H) \Gamma(H+1 / 2)}{\sin (\pi H) \Gamma(2 H) \Gamma(2-2 H)}=1 .
$$

Proof The arguments below use the well-known identity

$$
\Gamma(\alpha) \Gamma(1-\alpha)=\frac{\pi}{\sin (\pi \alpha)}, \quad \alpha \in(0,1)
$$

First let $H>1 / 2$. Applying (A.16) with $\alpha:=H-1 / 2$, it follows that

$$
\Gamma(3 / 2-H) \Gamma(H+1 / 2)=\frac{(H-1 / 2) \pi}{\sin (\pi(H-1 / 2))}=-\frac{(H-1 / 2) \pi}{\cos (\pi H)},
$$


because $\Gamma(H+1 / 2)=(H-1 / 2) \Gamma(H-1 / 2)$. Likewise,

$$
\begin{aligned}
\Gamma(2 H) \Gamma(2-2 H) & =(2 H-1) \Gamma(2 H-1) \Gamma(2-2 H) \\
& =\frac{(2 H-1) \pi}{\sin (2 \pi H-\pi)}=-\frac{(H-1 / 2) \pi}{\sin (\pi H) \cos (\pi H)},
\end{aligned}
$$

whence, as claimed, $\frac{\Gamma(3 / 2-H) \Gamma(H+1 / 2)}{\sin (\pi H) \Gamma(2 H) \Gamma(2-2 H)}=1$.

Now let $H<1 / 2$. Then applying (A.16) with $a:=1 / 2-H$ gives

$$
\begin{aligned}
\Gamma(3 / 2-H) \Gamma(H+1 / 2) & =(1 / 2-H) \Gamma(1 / 2-H) \Gamma(H+1 / 2) \\
& =\frac{(1 / 2-H) \pi}{\sin (\pi(1 / 2-H))}=\frac{(1 / 2-H) \pi}{\cos (\pi H)},
\end{aligned}
$$

and

$$
\Gamma(2 H) \Gamma(2-2 H)=\frac{\pi(1-2 H)}{\sin (2 \pi H)}=\frac{\pi(1 / 2-H)}{\sin (\pi H) \cos (\pi H)},
$$

whence the claim follows.

\section{A.4 Limit of the covariances}

Now for $s \neq t, s, t>0$ and $\zeta_{t}^{n}, \zeta_{s}^{n}$ as above in (A.13), consider the asymptotic covariance $R(s, t)=\lim _{n \rightarrow \infty} \Delta_{n}^{-2 H} E\left[\zeta_{t}^{n} \zeta_{s}^{n}\right]$, with

$$
\zeta_{t}^{n}=\tilde{c}_{H} \xi_{T\left(\kappa_{t}^{n}+1\right) / n, T \kappa_{t}^{n} / n},
$$

provided that the limit exists. The first lemma shows that this covariance vanishes asymptotically.

Lemma A.7 For any $s \neq t, s, t>0$, we have

$$
R(s, t)=\lim _{n \rightarrow \infty} \Delta_{n}^{-2 H} E\left[\zeta_{t}^{n} \zeta_{s}^{n}\right]=0
$$

Proof For $0<s<t$, the covariance is

$$
\begin{aligned}
E\left[\zeta_{t}^{n} \zeta_{s}^{n}\right] & =\tilde{c}_{H}^{2} E\left[\xi_{T\left(\kappa_{t}^{n}+1\right) / n, T \kappa_{t}^{n} / n} \xi_{\left.T\left(\kappa_{s}^{n}+1\right) / n, T \kappa_{s}^{n} / n\right]}\right. \\
= & \tilde{c}_{H}^{2} \int_{0}^{T \kappa_{s}^{n} / n} u^{1-2 H}\left(\int_{T \kappa_{s}^{n} / n}^{T\left(\kappa_{s}^{n}+1\right) / n} v^{H-1 / 2}(v-u)^{H-3 / 2} d v\right) \\
& \times\left(\int_{T \kappa_{t}^{n} / n}^{T\left(\kappa_{t}^{n}+1\right) / n} v^{H-1 / 2}(v-u)^{H-3 / 2} d v\right) d u .
\end{aligned}
$$


Recall that $T \kappa_{t}^{n} / n \uparrow t, T \kappa_{s}^{n} / n \uparrow s, T\left(\kappa_{t}^{n}+1\right) / n \downarrow t, T\left(\kappa_{s}^{n}+1\right) / n \downarrow s$ as $n \rightarrow \infty$. Thus

$$
\begin{aligned}
& \Delta_{n}^{-2 H} E\left[\zeta_{t}^{n} \zeta_{s}^{n}\right] \\
& \sim \tilde{c}_{H}^{2} s^{H-1 / 2} t^{H-1 / 2} \Delta_{n}^{-2 H} \int_{0}^{T \kappa_{s}^{n} / n} u^{1-2 H}\left(\int_{T \kappa_{s}^{n} / n}^{T\left(\kappa_{s}^{n}+1\right) / n}(v-u)^{H-3 / 2} d v\right) \\
& \times\left(\int_{T \kappa_{t}^{n} / n}^{T\left(\kappa_{t}^{n}+1\right) / n}(v-u)^{H-3 / 2} d v\right) d u \\
& =\tilde{c}_{H}^{2} s^{H-1 / 2} t^{H-1 / 2} \Delta_{n}^{2-2 H} \int_{0}^{T \kappa_{s}^{n} / n} u^{1-2 H}\left(\theta_{s}^{n}-u\right)^{H-3 / 2}\left(\theta_{t}^{n}-u\right)^{H-3 / 2} d u
\end{aligned}
$$

for some $\theta_{s}^{n} \in\left(T \kappa_{s}^{n} / n, T\left(\kappa_{s}^{n}+1\right) / n\right)$ and $\theta_{t}^{n} \in\left(T \kappa_{t}^{n} / n, T\left(\kappa_{t}^{n}+1\right) / n\right)$.

(i) Let $H>1 / 2$. The intuition is that for such $H$,

$$
\begin{aligned}
& \lim _{n \rightarrow \infty} \int_{0}^{T \kappa_{s}^{n} / n} u^{1-2 H}\left(\theta_{s}^{n}-u\right)^{H-3 / 2}\left(\theta_{t}^{n}-u\right)^{H-3 / 2} d u \\
& =\int_{0}^{s} u^{1-2 H}(s-u)^{H-3 / 2}(t-u)^{H-3 / 2} d u .
\end{aligned}
$$

To make this intuition rigorous, it remains to check that Lebesgue's dominated convergence theorem applies. To this end, notice that

$$
\begin{aligned}
& \int_{0}^{T \kappa_{s}^{n} / n} u^{1-2 H}\left(\theta_{s}^{n}-u\right)^{H-3 / 2}\left(\theta_{t}^{n}-u\right)^{H-3 / 2} d u \\
& =\left(T \frac{\kappa_{s}^{n}}{n}\right)^{-1} \int_{0}^{1} u^{1-2 H}\left(\frac{\theta_{s}^{n} n}{T \kappa_{s}^{n}}-u\right)^{H-3 / 2}\left(\frac{\theta_{t}^{n} n}{T \kappa_{s}^{n}}-u\right)^{H-3 / 2} d u,
\end{aligned}
$$

and

$$
\left(\frac{\theta_{s}^{n} n}{T \kappa_{s}^{n}}-u\right)^{H-3 / 2} \leq(1-u)^{H-3 / 2}, \quad\left(\frac{\theta_{t}^{n} n}{T \kappa_{s}^{n}}-u\right)^{H-3 / 2} \leq\left(\frac{t}{s+T / n}-u\right)^{H-3 / 2} .
$$

Note that for $n>\frac{2 T}{t-s}$,

$$
\frac{t}{s+T / n}>\frac{2 t}{s+t}, \quad\left(\frac{t}{s+T / n}-u\right)^{H-3 / 2}<\left(\frac{2 t}{s+t}-u\right)^{H-3 / 2},
$$

so that the integral

$$
\int_{0}^{1} u^{1-2 H}(1-u)^{H-3 / 2}\left(\frac{2 t}{s+t}-u\right)^{H-3 / 2} d u
$$

converges, thereby proving that for $H>1 / 2$,

$$
\lim _{n \rightarrow \infty} \Delta_{n}^{-2 H} E\left[\zeta_{s}^{n} \zeta_{t}^{n}\right]=0 .
$$


(ii) Turning to the case $H<1 / 2$, note that

$$
\begin{aligned}
\Delta_{n}^{-2 H} E\left[\zeta_{s}^{n} \zeta_{t}^{n}\right] \sim & (1 / 2-H)^{-2} \tilde{c}_{H}^{2} \Delta_{n}^{-2 H} s^{H-1 / 2} t^{H-1 / 2} \frac{\kappa_{s}^{n} T}{n} \\
& \times \int_{0}^{1} z^{1-2 H}\left(\left(\frac{1}{\kappa_{s}^{n}}+1-z\right)^{H-1 / 2}-(1-z)^{H-1 / 2}\right) \\
& \times\left(\left(\frac{\kappa_{t}^{n}+1}{\kappa_{s}^{n}}-z\right)^{H-1 / 2}-\left(\frac{\kappa_{t}^{n}}{\kappa_{s}^{n}}-z\right)^{H-1 / 2}\right) d z \\
\sim & \left.(1 / 2-H)^{-2} \tilde{c}_{H}^{2} s^{H+1 / 2} t^{H-1 / 2} \Delta_{n}^{-2 H}\right)^{1 / 2-H}-\left(1 / \kappa_{s}^{n}+1-z\right)^{1 / 2-H} \\
& \left.\times \int_{0}^{1} z^{1-2 H} \frac{(1-z)^{1 / 2-H}}{\left(1 / \kappa_{s}^{n}+1-z\right)^{1 / 2-H}(1-z)^{1 / 2-H}}\right) d z \\
= & \left(\frac{1}{2}-H\right)^{-2} \tilde{c}_{H}^{2} s^{H+1 / 2} t^{H-1 / 2} A_{n} .
\end{aligned}
$$

Observe for $0 \leq b \leq a$ and $q \in(0,1)$ the elementary inequality

$$
a^{q}-b^{q} \leq(a-b)^{q}
$$

Thus for $0 \leq z \leq 1$,

$$
\left|\left(1-z+\frac{1}{\kappa_{s}^{n}}\right)^{1 / 2-H}-(1-z)^{1 / 2-H}\right| \leq\left(\frac{1}{\kappa_{s}^{n}}\right)^{1 / 2-H} .
$$

As $T \kappa_{s}^{n} / n \uparrow s$, it follows that $1 / \kappa_{s}^{n}=O(1 / n)$. Note that

$$
\frac{1}{\left(1 / \kappa_{s}^{n}+1-z\right)^{1 / 2-H}}<\frac{1}{(1-z)^{1 / 2-H}}
$$

and

$$
\left(\frac{\kappa_{t}^{n}+1}{\kappa_{s}^{n}}-z\right)^{H-1 / 2}-\left(\frac{\kappa_{t}^{n}}{\kappa_{s}^{n}}-z\right)^{H-1 / 2}=\frac{H-1 / 2}{\kappa_{s}^{n}}\left(\theta_{s, t}^{n}-z\right)^{H-3 / 2}
$$

where $\theta_{s, t}^{n} \in\left(\kappa_{t}^{n} / \kappa_{s}^{n},\left(\kappa_{t}^{n}+1\right) / \kappa_{s}^{n}\right)$. Note also that for $n>\frac{2 T}{t-s}$,

$$
\theta_{s, t}^{n} \geq \kappa_{t}^{n} / \kappa_{s}^{n} \geq \frac{t}{s+T / n}>\frac{2 t}{s+t},
$$


whence $\left(\theta_{s, t}^{n}-z\right)^{H-3 / 2}<\left(\frac{t+s}{2 s}-1\right)^{H-3 / 2}$. From these considerations, it follows that

$$
\begin{aligned}
A_{n} & \leq \Delta_{n}^{-2 H}(O(1 / n))^{1 / 2-H} O(1 / n) \int_{0}^{1}(1-z)^{2 H-1} z^{1-2 H}\left(\theta_{s, t}^{n}-z\right)^{H-3 / 2} d z \\
& \leq \Delta_{n}^{-2 H}(O(1 / n))^{1 / 2-H} O(1 / n) \int_{0}^{1}(1-z)^{2 H-1} z^{1-2 H}\left(\frac{2 t}{s+t}-z\right)^{H-3 / 2} d z .
\end{aligned}
$$

Hence

$$
A_{n} \sim \Delta^{-2 H}\left(O(1 / n)^{1 / 2-H}\right) O(1 / n) \sim n^{2 H-1 / 2+H-1}=n^{3 H-3 / 2} \longrightarrow 0,
$$

and the statement follows.

\section{A.5 Limit of the value processes of the strategies $\pi(n)$}

Now consider the process $\eta_{t}^{n}:=\Delta_{n}^{-H} \zeta_{t}^{n} / \phi_{t}^{n}$, where

$$
\begin{aligned}
\phi_{t}^{n} & :=\Delta_{n}^{-2 H} \int_{T \kappa_{t}^{n} / n}^{T\left(\kappa_{t}^{n}+1\right) / n} Z_{H}^{2}\left(\frac{T\left(\kappa_{t}^{n}+1\right)}{n}, s\right) d s \\
& =\Delta_{n}^{-2 H} \operatorname{Var}_{T \kappa_{t}^{n} / n}\left[B_{T\left(\kappa_{t}^{n}+1\right) / n}^{H}-B_{T \kappa_{t}^{n} / n}^{H}\right] .
\end{aligned}
$$

Define a centered Gaussian process $\left(B_{t}\right)_{0 \leq t \leq T}$ by $B_{0}=0, E\left[B_{t} B_{s}\right]=0$ for $t \neq s$ and $E\left[B_{t}^{2}\right]=C_{H}, t \in(0, T]$, where

$$
C_{H}=1-\frac{\Gamma(3 / 2-H)}{\Gamma(H+1 / 2) \Gamma(2-2 H)} .
$$

Theorem A.8 For any $H \in(0,1) \backslash\{1 / 2\}$, as $n \rightarrow \infty$,

$$
\eta_{t}^{n} \Longrightarrow \frac{\Gamma(H+1 / 2) \Gamma(2-2 H)}{\Gamma(3 / 2-H)} B_{t},
$$

where $\Rightarrow$ denotes the weak convergence of probability laws.

Proof (i) Let $H>1 / 2$. Then

$$
\begin{aligned}
& \phi_{t}^{n} \\
& =\tilde{c}_{H}^{2} \Delta_{n}^{-2 H} \int_{T \kappa_{t}^{n} / n}^{T\left(\kappa_{t}^{n}+1\right) / n} s^{1-2 H}\left(\int_{s}^{T\left(\kappa_{t}^{n}+1\right) / n} u^{H-1 / 2}(u-s)^{H-3 / 2} d u\right)^{2} d s .
\end{aligned}
$$


As before, the limit of this expression is unchanged by omitting $s^{1-2 H}$ and $u^{H-1 / 2}$. Thus

$$
\begin{aligned}
\lim _{n \rightarrow \infty} \phi_{t}^{n} & =\frac{\tilde{c}_{H}^{2}}{(H-1 / 2)^{2}} \lim _{n \rightarrow \infty} \Delta_{n}^{-2 H} \int_{T \kappa_{t}^{n} / n}^{T\left(\kappa_{t}^{n}+1\right) / n}\left(\left(T \frac{\left(\kappa_{t}^{n}+1\right)}{n}-s\right)^{H-1 / 2}\right)^{2} d s \\
& =c_{H}^{2} \lim _{n \rightarrow \infty} \Delta_{n}^{-2 H} \int_{T \kappa_{t}^{n} / n}^{T\left(\kappa_{t}^{n}+1\right) / n}\left(T \frac{\left(\kappa_{t}^{n}+1\right)}{n}-s\right)^{2 H-1} d s \\
& =c_{H}^{2} \lim _{n \rightarrow \infty}\left(T \frac{\left(\kappa_{t}^{n}+1\right)}{n}-T \frac{\kappa_{t}^{n}}{n}\right)^{2 H} \frac{1}{2 H} \frac{n^{2 H}}{T^{2 H}} \\
& =\frac{c_{H}^{2}}{2 H}=\frac{\Gamma(3 / 2-H)}{\Gamma(H+1 / 2) \Gamma(2-2 H)},
\end{aligned}
$$

which yields the claim that $\eta_{t}^{n} \Rightarrow \frac{\Gamma(H+1 / 2) \Gamma(2-2 H)}{\Gamma(3 / 2-H)} B_{t}$.

(ii) In the case $H<1 / 2$, the limit equals

$$
\begin{aligned}
& \\
\lim _{n \rightarrow \infty} \phi_{t}^{n} & \\
=\lim _{n \rightarrow \infty} \Delta_{n}^{-2 H} c_{H}^{2} & \\
& \times \int_{T \kappa_{t}^{n} / n}^{T\left(\kappa_{t}^{n}+1\right) / n}\left(\left(T \frac{\left(\kappa_{t}^{n}+1\right)}{n}\right)^{H-1 / 2} s^{1 / 2-H}\left(T \frac{\left(\kappa_{t}^{n}+1\right)}{n}-s\right)^{H-1 / 2}\right. \\
& \quad-(H-1 / 2) s^{1 / 2-H} \\
& \left.\quad \times \int_{s}^{T\left(\kappa_{t}^{n}+1\right) / n} u^{H-3 / 2}(u-s)^{H-1 / 2} d u\right)^{2} d s .
\end{aligned}
$$

To evaluate this expression, first note that in view of the previous formulas,

$$
E\left[\left(B_{u}^{H}-B_{z}^{H}\right)^{2}\right]=\int_{0}^{z}\left(Z_{H}(u, s)-Z_{H}(z, s)\right)^{2} d s+\int_{z}^{u} Z_{H}^{2}(u, s) d s .
$$

Now let $z:=T \kappa_{t}^{n} / n$ and $u:=T\left(\kappa_{t}^{n}+1\right) / n$ so that $E\left[\left(B_{u}^{H}-B_{z}^{H}\right)^{2}\right]=(T / n)^{2 H}$. As already calculated,

$$
\begin{aligned}
\lim _{n \rightarrow \infty} \frac{1}{\Delta_{n}^{2 H}} \int_{0}^{z}\left(Z_{H}(u, s)-Z_{H}(z, s)\right)^{2} d s & =c_{H}^{2} \int_{0}^{\infty}\left((y+1)^{H-1 / 2}-y^{H-1 / 2}\right)^{2} d y \\
& =C_{H} .
\end{aligned}
$$

Therefore,

$$
\begin{aligned}
\lim _{n \rightarrow \infty} \phi_{t}^{n} & =\lim _{n \rightarrow \infty} \frac{1}{\Delta_{n}^{2 H}}\left(\frac{T}{n}\right)^{2 H}-1+\frac{\Gamma(3 / 2-H)}{\Gamma(H+1 / 2) \Gamma(2-2 H)} \\
& =\frac{\Gamma(3 / 2-H)}{\Gamma(H+1 / 2) \Gamma(2-2 H)},
\end{aligned}
$$

whence $\eta_{t}^{n} \Rightarrow \frac{\Gamma(H+1 / 2) \Gamma(2-2 H)}{\Gamma(3 / 2-H)} B_{t}$ also for $H<1 / 2$. 


\section{A.6 Final steps}

Proof of Theorem 2.2 For any $H \in(0,1) \backslash\{1 / 2\}$, as in the proof of Theorem A.8,

$$
\begin{aligned}
\lim _{n \rightarrow \infty} \frac{T}{n} E\left[R_{\gamma}\left(\pi(n), n, B^{H}\right)\right] & =\lim _{n \rightarrow \infty} \frac{T}{n} \sum_{k=0}^{n-1} E\left[\frac{E_{T k / n}^{2}\left[B_{T(k+1) / n}^{H}-B_{T k / n}^{H}\right]}{2 \gamma \operatorname{Var}_{T k / n}\left[B_{T(k+1) / n}^{H}-B_{T k / n}^{H}\right]}\right] \\
& =\lim _{n \rightarrow \infty} \frac{T}{n} \sum_{k=0}^{n-1} E\left[\frac{\Delta_{n}^{-2 H}\left(\zeta_{T k / n}^{n}\right)^{2}}{2 \gamma \phi_{T k / n}^{n}}\right] \\
& =\frac{\Gamma(H+1 / 2) \Gamma(2-2 H)}{2 \gamma \Gamma(3 / 2-H)} \int_{0}^{T} E\left[B_{t}^{2}\right] d t \\
& =\frac{T}{\gamma} C(H) \frac{\Gamma(H+1 / 2) \Gamma(2-2 H)}{2 \Gamma(3 / 2-H)} .
\end{aligned}
$$

The passage to the limit is justified by Lebesgue's theorem since the $E\left[\Delta_{n}^{-2 H}\left(\zeta_{T k / n}^{n}\right)^{2}\right]$ are bounded from above uniformly in $n, k$, and the $\phi_{T k / n}^{n}$ are bounded away from 0 uniformly in $n, k$, by Lemma A.9 below. Hence we obtain

$$
\begin{aligned}
V(H, \gamma) & =\lim _{n \rightarrow \infty} \frac{T}{n} E\left[R_{\gamma}\left(\pi(n), n, B^{H}\right)\right] \\
& =\frac{T}{\gamma}\left(\frac{\Gamma(H+1 / 2) \Gamma(2-2 H)}{2 \Gamma(3 / 2-H)}-\frac{1}{2}\right) .
\end{aligned}
$$

For $H<1 / 2$, an analogous argument yields (A.20) again, completing the proof.

Lemma A.9 The values $E\left[\Delta_{n}^{-2 H}\left(\zeta_{T k / n}^{n}\right)^{2}\right]$ are bounded from above uniformly in $n$, $k$, and the $\phi_{T k / n}^{n}$ are bounded away from 0 uniformly in $n, k$.

Proof First consider the case $H>1 / 2$. Note that $E\left[\Delta_{n}^{-2 H}\left(\zeta_{T k / n}^{n}\right)^{2}\right]$ is bounded from above by a constant multiple of $\Delta_{n}^{-2 H} I_{T\left(\kappa_{t}^{n}+1\right) / n, T \kappa_{t}^{n} / n}^{\sharp}$. For the right-hand side of (A.14), note that

$$
\begin{aligned}
\frac{\left(T \frac{\left(\kappa_{t}^{n}+1\right)}{n}\right)^{2 H-1} \Delta_{n}^{2-2 H}}{T \frac{\kappa_{t}^{n}}{n}} & =\frac{\kappa_{t}^{n}+1}{\kappa_{t}^{n}}\left(T \frac{\left(\kappa_{t}^{n}+1\right)}{n}\right)^{2 H-2} \Delta_{n}^{2-2 H} \\
& \leq 2\left(\frac{T}{n}\right)^{2 H-2} \Delta_{n}^{2-2 H} \leq 2
\end{aligned}
$$


Therefore,

$$
\begin{aligned}
& \Delta_{n}^{-2 H} I_{T\left(\kappa_{t}^{n}+1\right) / n, T \kappa_{t}^{n} / n}^{\sharp} \\
& \leq 2 \int_{0}^{1 / 2} s^{1-2 H}(1-s)^{2 H-3} d s \\
& \quad+\frac{2^{2 H-1} 2^{1-2 H}}{(H-1 / 2)^{2}} \int_{0}^{\infty}\left((y+1)^{H-1 / 2}-y^{H-1 / 2}\right)^{2} d y,
\end{aligned}
$$

and the right-hand side of (A.21) is constant. Furthermore, according to (A.17) and (A.18),

$$
\begin{aligned}
\phi_{t}^{n} & \geq \tilde{c}_{H}^{2} \Delta_{n}^{-2 H} \int_{T \kappa_{t}^{n} / n}^{T\left(\kappa_{t}^{n}+1\right) / n}\left(\int_{s}^{T\left(\kappa_{t}^{n}+1\right) / n}(u-s)^{H-3 / 2} d u\right)^{2} d s \\
& \geq c_{H}^{2} \Delta_{n}^{-2 H} \int_{T \kappa_{t}^{n} / n}^{T\left(\kappa_{t}^{n}+1\right) / n}\left(\frac{\left(\kappa_{t}^{n}+1\right) T}{n}-s\right)^{2 H-1} d s \\
& =\frac{\Gamma(3 / 2-H)}{\Gamma(H+1 / 2) \Gamma(2-2 H)} .
\end{aligned}
$$

Turning to the case $H<1 / 2$, observe that $\Delta_{n}^{-2 H} \tilde{I}_{T\left(\kappa_{t}^{n}+1\right) / n, T \kappa_{t}^{n} / n}^{\sharp}$ does not exceed

$$
\begin{aligned}
& \left(\frac{T}{n}\right)^{2 H-2} \Delta_{n}^{2-2 H} \int_{0}^{1 / 2} s^{1-2 H}(1-s)^{2 H-3} d s \\
& +\frac{1}{(H-1 / 2)^{2}} \int_{0}^{\infty}\left((y+1)^{H-1 / 2}-y^{H-1 / 2}\right)^{2} d y,
\end{aligned}
$$

which is a constant. Finally, it follows that

$$
\begin{aligned}
\phi_{t}^{n} \geq & \tilde{c}_{H}^{2} \Delta_{n}^{-2 H} \\
& \times \int_{T \kappa_{t}^{n} / n}^{T\left(\kappa_{t}^{n}+1\right) / n}\left(\left(T \frac{\left(\kappa_{t}^{n}+1\right)}{n}\right)^{H-1 / 2} s^{1 / 2-H}\left(T \frac{\left(\kappa_{t}^{n}+1\right)}{n}-s\right)^{H-1 / 2}\right)^{2} d s \\
\geq & \tilde{c}_{H}^{2} \Delta_{n}^{-2 H}\left(T \frac{\left(\kappa_{t}^{n}+1\right)}{n}\right)^{2 H-1}\left(T \frac{\kappa_{t}^{n}}{n}\right)^{1-2 H}\left(\frac{T}{n}\right)^{2 H} \frac{1}{2 H} \\
= & \frac{\tilde{c}_{H}^{2}}{2 H}\left(\frac{\kappa_{t}^{n}}{\kappa_{t}^{n}+1}\right)^{1-2 H} \geq \frac{\tilde{c}_{H}^{2}}{2 H}\left(\frac{1}{2}\right)^{1-2 H},
\end{aligned}
$$

which is also a positive constant.

\section{A.7 Trading costs}

Proof For any $1 \leq k \leq n-1$, we claim that $E\left[\left|\pi_{T(k+1) / n}(n)-\pi_{T k / n}(n)\right|^{\alpha}\right] \rightarrow \infty$ as $n \rightarrow \infty$, and we estimate its convergence rate. Indeed, $\pi_{T(k+1) / n}(n)-\pi_{T k / n}(n)$ is a 
Gaussian random variable; therefore it is sufficient to study

$$
E\left[\left(\pi_{T(k+1) / n}(n)-\pi_{T k / n}(n)\right)^{2}\right] .
$$

Recall that according to (2.5),

$$
\begin{aligned}
& \pi_{T(k+1) / n}(n)-\pi_{T k / n}(n) \\
& =\frac{E_{T(k+1) / n}\left[B_{T(k+2) / n}^{H}-B_{T(k+1) / n}^{H}\right]}{2 \operatorname{Var}_{T(k+1) / n}\left[B_{T(k+2) / n}^{H}-B_{T(k+1) / n}^{H}\right]} \\
& \quad-\frac{E_{T k / n}\left[B_{T(k+1) / n}^{H}-B_{T k / n}^{H}\right]}{2 \operatorname{Var}_{T k / n}\left[B_{T(k+1) / n}^{H}-B_{T k / n}^{H}\right]} \\
& =\tilde{c}_{H}\left(\frac{\int_{0}^{T(k+1) / n} s^{1 / 2-H}\left(\int_{T(k+1) / n}^{T(k+2) / n} v^{H-1 / 2}(v-s)^{H-3 / 2} d v\right) d W_{s}}{2 \operatorname{Var}_{T(k+1) / n}\left[B_{T(k+2) / n}^{H}-B_{T(k+1) / n}^{H}\right]}\right. \\
& \left.\quad+\frac{\int_{0}^{T k / n} s^{1 / 2-H}\left(\int_{T k / n}^{T(k+1) / n} v^{H-1 / 2}(v-s)^{H-3 / 2} d v\right) d W_{s}}{2 \operatorname{Var}_{T k / n}\left[B_{T(k+1) / n}^{H}-B_{T k / n}^{H}\right]}\right) .
\end{aligned}
$$

This expression contains in the first numerator the Gaussian random variable

$$
\int_{T k / n}^{T(k+1) / n} s^{1 / 2-H}\left(\int_{T(k+1) / n}^{T(k+2) / n} v^{H-1 / 2}(v-s)^{H-3 / 2} d v\right) d W_{s},
$$

which is independent from the other terms. Therefore,

$$
\begin{aligned}
& E\left[\left(\pi_{T(k+1) / n}(n)-\pi_{T k / n}(n)\right)^{2}\right] \\
& \geq \frac{\tilde{c}_{H}^{2}}{4} \frac{\int_{T k / n}^{T(k+1) / n} s^{1-2 H}\left(\int_{T(k+1) / n}^{T(k+2) / n} v^{H-1 / 2}(v-s)^{H-3 / 2} d v\right)^{2} d s}{\operatorname{Var}_{T(k+1) / n}^{2}\left[B_{T(k+2) / n}^{H}-B_{T(k+1) / n}^{H}\right]} .
\end{aligned}
$$

Without loss of generality, assume that $k \neq 0$, which implies that as $n \uparrow \infty$,

$$
\begin{aligned}
& \int_{T k / n}^{T(k+1) / n} s^{1-2 H}\left(\int_{T(k+1) / n}^{T(k+2) / n} v^{H-1 / 2}(v-s)^{H-3 / 2} d v\right)^{2} d s \\
& \sim \int_{T k / n}^{T(k+1) / n}\left(\int_{T(k+1) / n}^{T(k+2) / n}(v-s)^{H-3 / 2} d v\right)^{2} d s \\
& \sim n^{-2} \int_{T k / n}^{T(k+1) / n}\left(\theta_{n}-s\right)^{2 H-3} d s,
\end{aligned}
$$

where $\theta_{n} \in[T(k+1) / n, T(k+2) / n]$. The latter expression is

$$
\sim n^{-2}\left(\left(\theta_{n}-\frac{(k+1) T}{n}\right)^{2 H-2}-\left(\theta_{n}-T k / n\right)^{2 H-2}\right) .
$$


The function $x \mapsto(x-b)^{2 H-2}-(x-a)^{2 H-2}, a<b<x$, decreases in $x$ as its derivative is

$$
(2 H-2)\left((x-b)^{2 H-3}-(x-a)^{2 H-3}\right)<0 .
$$

Thus

$$
\begin{aligned}
& n^{-2}\left(\left(\theta_{n}-\frac{(k+1) T}{n}\right)^{2 H-2}-\left(\theta_{n}-T k / n\right)^{2 H-2}\right) \\
& \geq n^{-2}\left(\left(\frac{(k+2) T}{n}-\frac{(k+1) T}{n}\right)^{2 H-2}-\left(\frac{(k+2) T}{n}-T k / n\right)^{2 H-2}\right) \\
& =n^{-2}\left(n^{2-2 H}-\left(\frac{n}{2}\right)^{2-2 H}\right) T^{2 H-2} \sim n^{-2 H} .
\end{aligned}
$$

Furthermore, as shown in Sect. A.5,

$$
\operatorname{Var}_{T(k+1) / n}\left[B_{T(k+2) / n}^{H}-B_{T(k+1) / n}^{H}\right] \sim n^{-2 H} .
$$

Hence as $n \rightarrow \infty$, the right-hand side of (A.22) is of the order

$$
\frac{n^{-2 H}}{n^{-4 H}} \sim n^{2 H} .
$$

Proposition A.5 and Sect. A.5 imply also that for some $C>0$,

$$
E\left[\pi_{T(k+1) / n}^{2}(n)\right] \leq C n^{2 H} \quad \text { for all } k, n,
$$

and hence $E\left[\left(\pi_{T(k+1) / n}(n)-\pi_{T k / n}(n)\right)^{2}\right] \sim n^{2 H}$ follows. Thus

$$
E\left[\sum_{k=0}^{n-1} \lambda\left|\pi_{T(k+1) / n}(n)-\pi_{T k / n}(n)\right|^{\alpha}\right]=O\left(n^{1+\alpha H}\right) .
$$

\section{A.8 Convergence}

As the Gaussian process $B$ is a white noise, it is unlikely that its approximations $\eta_{t}^{n}$ may converge other than in law. Consider the step functions defined earlier in (A.13),

$$
\zeta_{t}^{n}=\tilde{c}_{H} \xi_{T\left(\kappa_{t}^{n}+1\right) / n, T \kappa_{t}^{n} / n},
$$

and in order to simplify calculations, focus on the dyadic partitions of $[0, T]$.

Let the point $t \in(0, T)$ be fixed as before. Denote $\kappa_{t}^{2^{n}}:=\left\lfloor\frac{2^{n} t}{T}\right\rfloor$. Then

$$
T \frac{\kappa_{t}^{2^{n}}}{2^{n}} \leq t \leq T \frac{\left(\kappa_{t}^{2^{n}}+1\right)}{2^{n}}
$$

Introducing the notation

$$
t_{n}:=T \frac{\kappa_{t}^{2^{n}}}{2^{n}}, \quad t_{n}^{\prime}=t_{n}+\frac{T}{2^{n}}, \quad \Delta_{n}:=T / 2^{n},
$$


if we establish that the expectation

$$
E\left[\left(\left(\Delta_{n}\right)^{-H} \zeta_{t}^{2^{n}}-\left(\Delta_{n+1}\right)^{-H} \zeta_{t}^{2^{n+1}}\right)^{2}\right]
$$

does not converge to 0 as $n \rightarrow \infty$, then $L^{2}$-convergence cannot take place in Theorem A.5 above. We consider two cases, depending on the location of $t$.

Theorem A.10 The limit

$$
\lim _{n \rightarrow \infty} E\left[\left(\left(\Delta_{n}\right)^{-H} \zeta_{t}^{2^{n}}-\left(\Delta_{n+1}\right)^{-H} \zeta_{t}^{2^{n+1}}\right)^{2}\right]
$$

exists and is nonzero.

Proof (i) Let $t \in\left[t_{n},\left(t_{n}+t_{n}^{\prime}\right) / 2\right]$. Note that in this case, $t_{n+1}=t_{n}, t_{n+1}^{\prime}=\left(t_{n}+t_{n}^{\prime}\right) / 2$. Then

$$
\zeta_{t}^{2^{n+1}}=\tilde{c}_{H} \xi_{t_{n+1}^{\prime}, t_{n+1}}=\tilde{c}_{H} \xi_{\left(t_{n}+t_{n}^{\prime}\right) / 2, t_{n}} .
$$

(ii) Let $t \in\left[\left(t_{n}+t_{n}^{\prime}\right) / 2, t_{n}^{\prime}\right]=\left[t_{n+1}, t_{n+1}^{\prime}\right]$. Then

$$
\zeta_{t}^{2^{n+1}}=\tilde{c}_{H} \xi_{t_{n}^{\prime}, \frac{t_{n}+t_{n}^{\prime}}{2}}
$$

Consider the difference of $\left(\Delta_{n}\right)^{-H} \zeta_{t}^{2^{n}}$ and $\left(\Delta_{n+1}\right)^{-H} \zeta_{t}^{2^{n+1}}$ in both cases (i) and (ii), up to a constant multiplier $\tilde{c}_{H}$ that can and will be omitted.

In case (i), $\Delta_{n+1}^{-H} \zeta_{t}^{2^{n+1}}-\Delta_{n}^{-H} \zeta_{t}^{2^{n}}$ is asymptotically equivalent to

$$
\Delta_{n+1}^{-H} \xi_{\left(t_{n}+t_{n}^{\prime}\right) / 2, t_{n}}-\Delta_{n}^{-H} \xi_{t_{n}^{\prime}, t_{n}} \text {. }
$$

Consider

$$
\begin{array}{rl}
E & {\left[\left(\Delta_{n+1}^{-H} \xi_{\left(t_{n}+t_{n}^{\prime}\right) / 2, t_{n}}-\Delta_{n}^{-H} \xi_{t_{n}^{\prime}, t_{n}}\right)^{2}\right]} \\
=E & E\left[\Delta_{n+1}^{-H} \int_{0}^{t_{n}} s^{1 / 2-H}\left(\int_{t_{n}}^{\left(t_{n}+t_{n}^{\prime}\right) / 2} v^{H-1 / 2}(v-s)^{H-3 / 2} d v\right) d W_{s}\right. \\
& \left.\left.-\Delta_{n}^{-H} \int_{0}^{t_{n}} s^{1 / 2-H}\left(\int_{t_{n}}^{t_{n}^{\prime}} v^{H-1 / 2}(v-s)^{H-3 / 2} d v\right) d W_{s}\right)^{2}\right] \\
= & \Delta_{n+1}^{-2 H} \int_{0}^{t_{n}} s^{1-2 H}\left(\int_{t_{n}}^{\left(t_{n}+t_{n}^{\prime}\right) / 2} v^{H-1 / 2}(v-s)^{H-3 / 2} d v\right)^{2} d s \\
& -\frac{2}{\Delta_{n}^{H} \Delta_{n+1}^{H}} \int_{0}^{t_{n}} s^{1-2 H}\left(\int_{t_{n}}^{\left(t_{n}+t_{n}^{\prime}\right) / 2} v^{H-1 / 2}(v-s)^{H-3 / 2} d v\right) \\
& +\Delta_{n}^{-2 H} \int_{0}^{t_{n}} s^{1-2 H}\left(\int_{t_{n}}^{t_{n}^{\prime}} v^{H-1 / 2}(v-s)^{H-3 / 2} d v\right)^{2} d s \\
=: & I_{1}^{n}-2 I_{2}^{n}+I_{3}^{n} \cdot
\end{array}
$$


Here $I_{1}^{n}$ and $I_{3}^{n}$ are evaluated like $\zeta_{t}^{n}$ in Proposition A.5. For completeness, we repeat the main steps for $I_{1}^{n}$, as they are similar for $I_{3}^{n}$.

As $t_{n} \leq v \leq\left(t_{n}+t_{n}^{\prime}\right) / 2$ and $t_{n} \rightarrow t, t_{n}^{\prime} \rightarrow t$, the asymptotic behaviour of $I_{1}^{n}$ is the same as for the simpler integral

$$
\begin{aligned}
\tilde{I}_{1}^{n} & :=\frac{t_{n}^{2 H-1}}{\Delta_{n+1}^{2 H}} \int_{0}^{t_{n}} s^{1-2 H}\left(\int_{t_{n}}^{\left(t_{n}+t_{n}^{\prime}\right) / 2}(v-s)^{H-3 / 2} d v\right)^{2} d s \\
& =\frac{1}{(H-1 / 2)^{2}} \frac{t_{n}^{2 H-1}}{\Delta_{n+1}^{2 H}} \int_{0}^{t_{n}} s^{1-2 H}\left(\left(\frac{t_{n}+t_{n}^{\prime}}{2}-s\right)^{H-1 / 2}-\left(t_{n}-s\right)^{H-1 / 2}\right)^{2} d s \\
& =\frac{1}{(H-1 / 2)^{2}} \frac{t_{n}^{2 H}}{\Delta_{n+1}^{2 H}} \int_{0}^{1} s^{1-2 H}\left(\left(\frac{1}{2}+\frac{t_{n}^{\prime}}{2 t_{n}}-s\right)^{H-1 / 2}-(1-s)^{H-1 / 2}\right)^{2} d s \\
& =\frac{1}{(H-1 / 2)^{2}} \frac{t_{n}^{2 H}}{\Delta_{n+1}^{2 H}}\left(\int_{0}^{1 / 2}+\int_{1 / 2}^{1}\right) .
\end{aligned}
$$

The integral $\int_{0}^{1 / 2}$ will be transformed as

$$
\int_{1 / 2}^{1}(1-x)^{1-2 H}\left(\left(\frac{1}{2}\left(\frac{t_{n}^{\prime}}{t_{n}}-1\right)+x\right)^{H-1 / 2}-x^{H-1 / 2}\right)^{2} d x .
$$

Furthermore,

$$
\left(\frac{1}{2}\left(\frac{t_{n}^{\prime}}{t_{n}}-1\right)+x\right)^{H-1 / 2}-x^{H-1 / 2}=\frac{1}{2} \frac{t_{n}^{\prime}-t_{n}}{t_{n}}(\delta+x)^{H-3 / 2}(H-1 / 2),
$$

where $0<\delta<\frac{1}{2} \frac{t_{n}^{\prime}-t_{n}}{t_{n}}$ and $\delta+x \sim x$. Note that

$$
\frac{t_{n}^{\prime}-t_{n}}{t_{n}}=\frac{T}{2 n} \frac{1}{t_{n}} \longrightarrow 0
$$

and therefore $\int_{0}^{1 / 2} \sim\left(\frac{t_{n}^{\prime}-t_{n}}{t_{n}}\right)^{2}$ so that $\Delta_{n+1}^{-2 H} \int_{0}^{1 / 2} \rightarrow 0$ as $n \rightarrow \infty$.

For $t_{n}^{2 H}(H-1 / 2)^{-2} \Delta_{n+1}^{-2 H} \int_{1 / 2}^{1}$, the change of variable $x=\frac{t_{n}^{\prime}-t_{n}}{2 t_{n}} y=\frac{T}{2^{n+1} t_{n}} y$ yields

$$
\begin{aligned}
& \int_{1 / 2}^{1} s^{1-2 H}\left(\left(\frac{1}{2}+\frac{t_{n}^{\prime}}{2 t_{n}}-s\right)^{H-1 / 2}-(1-s)^{H-1 / 2}\right)^{2} d s \\
& =\int_{0}^{1 / 2}(1-x)^{1-2 H}\left(\left(\frac{t_{n}^{\prime}-t_{n}}{2 t_{n}}+x\right)^{H-1 / 2}-x^{H-1 / 2}\right)^{2} d x \\
& =\left(\frac{T}{2^{n+1} t_{n}}\right)^{2 H} \int_{0}^{2^{n} t_{n} / T}\left(1-\frac{T}{2^{n+1} t_{n}} y\right)^{1-2 H}\left((1+y)^{H-1 / 2}-y^{H-1 / 2}\right) d y .
\end{aligned}
$$


Therefore

$$
\tilde{I}_{1}^{n} \sim \frac{1}{(H-1 / 2)^{2}} \int_{0}^{\infty}\left((1+y)^{H-1 / 2}-y^{H-1 / 2}\right)^{2} d y .
$$

(This is the same result as in the argument leading to Proposition A.5 above.)

Concerning $I_{3}^{n}$, similar calculations lead to

$$
\begin{aligned}
I_{3}^{n} \sim \tilde{I}_{3}^{n} & =\frac{t_{n}^{2 H-1}}{\Delta_{n}^{2 H}(H-1 / 2)^{2}} \int_{0}^{t_{n}} s^{1-2 H}\left(\left(t_{n}^{\prime}-s\right)^{H-1 / 2}-\left(t_{n}-s\right)^{H-1 / 2}\right)^{2} d s \\
& =\frac{t_{n}^{2 H-1}}{\Delta_{n}^{2 H}(H-1 / 2)^{2}} \int_{0}^{1} s^{1-2 H}\left(\left(\frac{t_{n}^{\prime}}{t_{n}}-s\right)^{H-1 / 2}-(1-s)^{H-1 / 2}\right)^{2} d s \\
& =\frac{t_{n}^{2 H-1}}{\Delta_{n}^{2 H}(H-1 / 2)^{2}}\left(\int_{0}^{1 / 2}+\int_{1 / 2}^{1}\right) .
\end{aligned}
$$

The term coming from the first integral, as always, tends to 0 . The term coming from the second integral is equivalent to

$$
\begin{aligned}
& \frac{t_{n}^{2 H}\left(t_{n}^{\prime}-t_{n}\right)^{2 H}}{\Delta_{n}^{2 H} t_{n}^{2 H}(H-1 / 2)^{2}} \int_{0}^{\infty}\left((y+1)^{H-1 / 2}-y^{H-1 / 2}\right)^{2} d y \\
& \sim \frac{1}{(H-1 / 2)^{2}} \int_{0}^{\infty}\left((y+1)^{H-1 / 2}-y^{H-1 / 2}\right)^{2} d y .
\end{aligned}
$$

(This is the same result as for $I_{1}^{n}$.) Now consider

$$
\begin{aligned}
I_{2}^{n}= & \frac{1}{\Delta_{n}^{H} \Delta_{n+1}^{H}} \int_{0}^{t_{n}} s^{1-2 H}\left(\int_{t_{n}}^{\left(t_{n}+t_{n}^{\prime}\right) / 2} v^{H-1 / 2}(v-s)^{H-3 / 2} d v\right) \\
& \times\left(\int_{t_{n}}^{t_{n}^{\prime}} v^{H-1 / 2}(v-s)^{H-3 / 2} d v\right) d s \\
\sim & \frac{2^{H} t_{n}^{2 H-1}}{\Delta_{n}^{2 H}(H-1 / 2)^{2}} \int_{0}^{t_{n}} s^{1-2 H}\left(\left(\frac{t_{n}+t_{n}^{\prime}}{2}-s\right)^{H-1 / 2}-\left(t_{n}-s\right)^{H-1 / 2}\right) \\
= & \frac{2^{H} t_{n}^{2 H}}{\Delta_{n}^{2 H}(H-1 / 2)^{2}} \int_{0}^{1} s^{1-2 H}\left(\left(\frac{1}{2}+\frac{t_{n}^{\prime}}{2 t_{n}}-s\right)^{H-1 / 2}-(1-s)^{H-1 / 2}\right) \\
& =\frac{2^{H} t_{n}^{2 H}}{\Delta_{n}^{2 H}(H-1 / 2)^{2}}\left(\int_{0}^{1 / 2}+\int_{1 / 2}^{1}\right) .
\end{aligned}
$$


The term coming from the first integral tends to 0 as $n \rightarrow \infty$ because it is equivalent to $\Delta_{n}^{2} / \Delta_{n}^{2 H}$. Using the changes of variables $s=1-x$ and $x=\frac{t_{n}^{\prime}-t_{n}}{2 t_{n}} y$, it follows that

$$
\begin{aligned}
& \frac{2^{H} t_{n}^{2 H}}{\Delta_{n}^{2 H}(H-1 / 2)^{2}} \int_{1 / 2}^{1} s^{1-2 H}\left(\left(\frac{1}{2}+\frac{t_{n}^{\prime}}{t_{n}}-s\right)^{H-1 / 2}-(1-s)^{H-1 / 2}\right) \\
& \times\left(\left(\frac{t_{n}^{\prime}}{t_{n}}-s\right)^{H-1 / 2}-(1-s)^{H-1 / 2}\right) d s \\
& =\frac{2^{H} t_{n}^{2 H}}{\Delta_{n}^{2 H}(H-1 / 2)^{2}} \int_{0}^{1 / 2}(1-x)^{1-2 H}\left(\left(\frac{-t_{n}+t_{n}^{\prime}}{2 t_{n}}+x\right)^{H-1 / 2}-x^{H-1 / 2}\right) \\
& \times\left(\left(\frac{t_{n}^{\prime}-t_{n}}{t_{n}}+x\right)^{H-1 / 2}-x^{H-1 / 2}\right) d x \\
& =\frac{2^{H} t_{n}^{2 H}}{\Delta_{n}^{2 H}(H-1 / 2)^{2}}\left(\frac{t_{n}^{\prime}-t_{n}}{2 t_{n}}\right)^{2 H} \\
& \times \int_{0}^{t_{n} /\left(t_{n}^{\prime}-t_{n}\right)}\left(1-\frac{t_{n}^{\prime}-t_{n}}{2 t_{n}} y\right)^{1-2 H}\left((1+y)^{H-1 / 2}-y^{H-1 / 2}\right) \\
& \times\left((2+y)^{H-1 / 2}-y^{H-1 / 2}\right) d y \\
& \longrightarrow \frac{2^{-H}}{(H-1 / 2)^{2}} \int_{0}^{\infty}\left((1+y)^{H-1 / 2}-y^{H-1 / 2}\right)\left((2+y)^{H-1 / 2}-y^{H-1 / 2}\right) d y \text {. }
\end{aligned}
$$

Therefore the limit equals

$$
\begin{aligned}
& \frac{2}{(H-1 / 2)^{2}} \int_{0}^{\infty}\left((y+1)^{H-1 / 2}-y^{H-1 / 2}\right)^{2} d y \\
& -2 \frac{2^{-H}}{(H-1 / 2)^{2}} \int_{0}^{\infty}\left((y+1)^{H-1 / 2}-y^{H-1 / 2}\right)\left((y+2)^{H-1 / 2}-y^{H-1 / 2}\right) d y .
\end{aligned}
$$

The change of variable $y=z / 2$ yields

$$
\begin{aligned}
\int_{0}^{\infty}\left((y+1)^{H-1 / 2}-y^{H-1 / 2}\right)^{2} d y & =\frac{1}{2} \int_{0}^{\infty}\left(\left(\frac{z}{2}-1\right)^{H-1 / 2}-\left(\frac{z}{2}\right)^{H-1 / 2}\right) d z \\
& =\frac{1}{2^{2 H}} \int_{0}^{\infty}\left((z+2)^{H-1 / 2}-z^{H-1 / 2}\right)^{2} d z
\end{aligned}
$$


and therefore the limit becomes

$$
\begin{aligned}
& \frac{1}{(H-1 / 2)^{2}}\left(\int_{0}^{\infty}\left((y+1)^{H-1 / 2}-y^{H-1 / 2}\right)^{2} d y\right. \\
& +\frac{1}{2^{2 H}} \int_{0}^{\infty}\left((y+2)^{H-1 / 2}-y^{H-1 / 2}\right)^{2} d y \\
& \left.\quad-2^{1-H} \int_{0}^{\infty}\left((y+1)^{H-1 / 2}-y^{H-1 / 2}\right)\left((y+2)^{H-1 / 2}-y^{H-1 / 2}\right) d y\right) \\
& =\frac{1}{(H-1 / 2)^{2}} \int_{0}^{\infty}\left((y+1)^{H-1 / 2}-y^{H-1 / 2}\right. \\
& \left.\quad-\frac{1}{2^{H}}(y+2)^{H-1 / 2}+\frac{1}{2^{H}} y^{H-1 / 2}\right)^{2} d y
\end{aligned}
$$

$\neq 0$.

Let $t=T j_{0} / 2^{m_{0}}$ for some $j_{0} \geq 1, m_{0} \geq 1$. Then $t \in\left[t_{n}, t_{n}^{\prime}\right]$ for $n \geq m_{0}$, i.e., case (i) holds. Therefore at least for $t$ of the form $T j / 2^{m}$, we have no $L^{2}$ convergence of $\zeta_{t}^{2^{n}}$. If $t$ is not of this form, then the situation will switch from (i) to (ii).

Consider now case (ii). Then $\Delta_{t}^{n}=\Delta_{n+1}^{-H} \zeta_{t}^{2^{n+1}}-\Delta_{n}^{-H} \zeta_{t}^{2^{n}}$ and $\Delta_{t}^{n}$ has the same limit (in the mean-square sense) as $\Delta_{n+1}^{-H} \xi_{t_{n}^{\prime}},\left(t_{n}+t_{n}^{\prime}\right) / 2-\Delta_{n}^{-H} \xi_{t_{n}^{\prime}, t_{n}}$. Consider

$$
\begin{aligned}
& E\left[\left(\Delta_{n+1}^{-H} \xi_{t_{n}^{\prime},\left(t_{n}+t_{n}^{\prime}\right) / 2}-\Delta_{n}^{-H} \xi_{t_{n}^{\prime}, t_{n}}\right)^{2}\right] \\
& =E\left[\left(\Delta_{n+1}^{-H} \int_{0}^{\left(t_{n}+t_{n}^{\prime}\right) / 2} s^{1 / 2-H}\left(\int_{\left(t_{n}+t_{n}^{\prime}\right) / 2}^{t_{n}^{\prime}} v^{H-1 / 2}(v-s)^{H-3 / 2} d v\right) d W_{s}\right.\right. \\
& \left.\left.-\Delta_{n}^{-H} \int_{0}^{t_{n}} s^{1 / 2-H}\left(\int_{t_{n}}^{t_{n}^{\prime}} v^{H-1 / 2}(v-s)^{H-3 / 2}\right) d W_{s}\right)^{2}\right] \\
& =\Delta_{n+1}^{-2 H} \int_{0}^{\left(t_{n}+t_{n}^{\prime}\right) / 2} s^{1-2 H}\left(\int_{\left(t_{n}+t_{n}^{\prime}\right) / 2}^{t_{n}^{\prime}} v^{H-1 / 2}(v-s)^{H-3 / 2} d v\right)^{2} d s \\
& -\frac{2}{\Delta_{n}^{H} \Delta_{n+1}^{H}} \int_{0}^{t_{n}} s^{1-2 H}\left(\int_{\left(t_{n}+t_{n}^{\prime}\right) / 2}^{t_{n}^{\prime}} v^{H-1 / 2}(v-s)^{H-3 / 2} d v\right) \\
& \times\left(\int_{t_{n}}^{t_{n}^{\prime}} v^{H-1 / 2}(v-s)^{H-3 / 2} d v\right) d s \\
& +\Delta_{n}^{-2 H} \int_{0}^{t_{n}} s^{1-2 H}\left(\int_{t_{n}}^{t_{n}^{\prime}} v^{H-1 / 2}(v-s)^{H-3 / 2} d v\right)^{2} d s \\
& =: I_{1}^{n}-2 I_{2}^{n}+I_{3}^{n} \text {. }
\end{aligned}
$$

As before, the limit of each term does not change when we replace $v^{H-1 / 2}$ by $t^{H-1 / 2}$. Therefore, setting $1-s=x, x=\frac{t_{n}^{\prime}-t_{n}}{2 t_{n}} y$ and noting $\left(t_{n}^{\prime}-t_{n}\right) / 2=T / 2^{n+1}=\Delta_{n+1}$, 
it follows that

$$
\begin{aligned}
I_{1}^{n} \sim & \frac{t^{2 H-1}}{\Delta_{n+1}^{2 H}(H-1 / 2)^{2}} \int_{0}^{t_{n}} s^{1-2 H}\left(\left(t_{n}^{\prime}-s\right)^{H-1 / 2}-\left(\frac{t_{n}^{\prime}+t_{n}}{2}-s\right)^{H-1 / 2}\right)^{2} d s \\
\sim & \frac{t^{2 H}}{\Delta_{n+1}^{2 H}(H-1 / 2)^{2}} \\
& \times \int_{0}^{1} s^{1-2 H}\left(\left(\frac{t_{n}^{\prime}-t_{n}}{t_{n}}+1-s\right)^{H-1 / 2}-\left(\frac{t_{n}^{\prime}-t_{n}}{2 t_{n}}+1-s\right)^{H-1 / 2}\right)^{2} d s \\
= & \frac{t^{2 H}}{\Delta_{n+1}^{2 H}(H-1 / 2)^{2}}\left(\int_{0}^{1 / 2}+\int_{1 / 2}^{1}\right) \\
\sim & \frac{t^{2 H}}{\Delta_{n+1}^{2 H}(H-1 / 2)^{2}}\left(\frac{t_{n}^{\prime}-t_{n}}{2 t_{n}}\right)^{2 H} \\
& \times \int_{0}^{t_{n} /\left(t_{n}^{\prime}-t_{n}\right)}\left(1-\frac{t_{n}^{\prime}-t_{n}}{2 t_{n}} y\right)^{1-2 H}\left((y+2)^{H-1 / 2}-(y+1)^{H-1 / 2}\right)^{2} d y \\
\sim & \frac{1}{(H-1 / 2)^{2}} \int_{0}^{\infty}\left((2+y)^{H-1 / 2}-(1+y)^{H-1 / 2}\right)^{2} d y .
\end{aligned}
$$

Similarly,

$$
I_{3}^{n} \sim \frac{1}{(H-1 / 2)^{2}} \int_{0}^{\infty}\left((y+1)^{H-1 / 2}-y^{H-1 / 2}\right)^{2} d y
$$

Now,

$$
\begin{aligned}
& I_{2}^{n} \sim \frac{t_{n}^{2 H}}{(H-1 / 2)^{2} \Delta_{n}^{H} \Delta_{n+1}^{H}} \int_{1 / 2}^{1} s^{1-2 H}\left(\left(\frac{t_{n}^{\prime}}{t_{n}}-s\right)^{H-1 / 2}-\left(\frac{t_{n}+t_{n}^{\prime}}{2 t_{n}}-s\right)^{H-1 / 2}\right) \\
& \times\left(\left(\frac{t_{n}^{\prime}}{t_{n}}-s\right)^{H-1 / 2}-(1-s)^{H-1 / 2}\right) d s \\
& \sim \frac{\left(\frac{t_{n}^{\prime}-t_{n}}{2}\right)^{2 H}}{(H-1 / 2)^{2} \Delta_{n}^{H} \Delta_{n+1}^{H}} \int_{0}^{\infty}\left((y+2)^{H-1 / 2}-(y+1)^{H-1 / 2}\right) \\
& \times\left((y+2)^{H-1 / 2}-y^{H-1 / 2}\right) d y \\
& \sim \frac{2^{-H}}{(H-1 / 2)^{2}} \int_{0}^{\infty}\left((y+2)^{H-1 / 2}-(y+1)^{H-1 / 2}\right) \\
& \times\left((y+2)^{H-1 / 2}-y^{H-1 / 2}\right) d y .
\end{aligned}
$$

Rewrite now $I_{3}^{n}$ (using the substitution $y=v / 2$ ) as

$$
I_{3}^{n} \sim \frac{2^{-2 H}}{(H-1 / 2)^{2}} \int_{0}^{\infty}\left((y+2)^{H-1 / 2}-y^{H-1 / 2}\right)^{2} d y .
$$


In summary, it follows that

$$
\begin{aligned}
& E\left[\left(\frac{1}{\Delta_{n+1}^{H}} \xi_{t_{n}^{\prime},\left(t_{n}+t_{n}^{\prime}\right) / 2}-\frac{1}{\Delta_{n}^{H}} \xi_{t_{n}^{\prime}, t_{n}}\right)^{2}\right] \\
& \sim \frac{1}{(H-1 / 2)^{2}} \int_{0}^{\infty}\left((2+y)^{H-1 / 2}-(1+y)^{H-1 / 2}\right. \\
& \left.\quad-2^{-H}\left((2+y)^{H-1 / 2}-y^{H-1 / 2}\right)\right)^{2} d y>0
\end{aligned}
$$

which shows that $L^{2}$-convergence also does not hold in case (ii).

Remark A.11 An inspection of the above proof shows that the limits in (A.19) and (A.23) remain the same if $\mathrm{fBm}$ is replaced by an $\mathrm{fBm}$ with drift, which corresponds to adding to $\zeta_{t}^{n}$ a term proportional to $\Delta_{n}$. Repeating the same calculations in that setting, it turns out that because $\Delta_{n}^{-2 H} \Delta_{n}^{2}=\Delta_{n}^{-2 H+2}$ vanishes as $n$ increases to infinity (since $H \in(0,1))$, the extra term is inconsequential.

\section{References}

1. Baillie, R.T.: Long memory processes and fractional integration in econometrics. J. Econom. 73, 5-59 (1996)

2. Cheridito, P.: Arbitrage in fractional Brownian motion models. Finance Stoch. 7, 533-553 (2003)

3. Czichowsky, C., Peyre, R., Schachermayer, W., Yang, J.: Shadow prices, fractional Brownian motion, and portfolio optimisation under transaction costs. Finance Stoch. 22, 161-180 (2018)

4. Czichowsky, C., Schachermayer, W.: Portfolio optimisation beyond semimartingales: shadow prices and fractional Brownian motion. Ann. Appl. Probab. 27, 1414-1451 (2017)

5. Dasgupta, A., Kallianpur, G.: Arbitrage opportunities for a class of Gladyshev processes. Appl. Math. Optim. 41, 377-385 (2000)

6. Fama, E.F., French, K.R.: Permanent and temporary components of stock prices. J. Polit. Econ. 96, 246-273 (1988)

7. Greene, M.T., Fielitz, B.D.: Long-term dependence in common stock returns. J. Financ. Econ. 4, 339-349 (1977)

8. Guasoni, P.: No arbitrage under transaction costs, with fractional Brownian motion and beyond. Math. Finance 16, 569-582 (2006)

9. Guasoni, P., Nika, Z., Rásonyi, M.: Trading fractional Brownian motion. SIAM J. Financ. Math. 10, 769-789 (2019)

10. Guasoni, P., Rásonyi, M., Schachermayer, W.: Consistent price systems and face-lifting pricing under transaction costs. Ann. Appl. Probab. 18, 491-520 (2008)

11. Guasoni, P., Weber, M.H.: Nonlinear price impact and portfolio choice. Math. Finance 30, 341-376 (2020)

12. Jacobsen, B.: Long term dependence in stock returns. J. Empir. Finance 3, 393-417 (1996)

13. Lo, A.W.: Long-term memory in stock market prices. Econometrica 59, 1279-1313 (1991)

14. Mandelbrot, B.B.: When can price be arbitraged efficiently? A limit to the validity of the random walk and martingale models. Rev. Econ. Stat. 53, 225-236 (1971)

15. Mishura, Y.S.: Stochastic Calculus for Fractional Brownian Motion and Related Processes. Lecture Notes in Mathematics, vol. 1929. Springer, Berlin (2008)

16. Norros, I., Valkeila, E., Virtamo, J.: An elementary approach to a Girsanov formula and other analytical results on fractional Brownian motions. Bernoulli 5, 571-587 (1999)

17. Poterba, J.M., Summers, L.H.: Mean reversion in stock prices: evidence and implications. J. Financ. Econ. 22, 27-59 (1988)

18. Revuz, D., Yor, M.: Continuous Martingales and Brownian Motion, 3rd edn. Springer, Berlin (1999) 
19. Rogers, L.C.G.: Arbitrage with fractional Brownian motion. Math. Finance 7, 95-105 (1997)

20. Salopek, D.M.: Tolerance to arbitrage. Stoch. Process. Appl. 76, 217-230 (1998)

21. Sun, Z., Qin, H.: Some results on the derivatives of the gamma and incomplete gamma function for non-positive integers. IAENG Int. J. Appl. Math. 47, 265-270 (2017)

22. Teverovsky, V., Taqqu, M.S., Willinger, W.: A critical look at Lo's modified R/S statistic. J. Stat. Plan. Inference 80, 211-227 (1999)

23. Willinger, W., Taqqu, M.S., Teverovsky, V.: Stock market prices and long-range dependence. Finance Stoch. 3, 1-13 (1999) 